\title{
Fast and accurate elastic analysis of laminated composite plates via isogeometric collocation and an equilibrium-based stress recovery approach
}

\author{
Alessia Patton $^{\mathrm{a}, *}$, John-Eric Dufour ${ }^{\mathrm{b}}$, Pablo Antolin ${ }^{\mathrm{c}}$, Alessandro Reali ${ }^{\mathrm{a}, \mathrm{d}}$ \\ ${ }^{a}$ Department of Civil Engineering and Architecture - University of Pavia \\ Via Ferrata, 3, 27100 Pavia, Italy \\ ${ }^{b}$ Mechanical and Aerospace Engineering Department \\ University of Texas at Arlington \\ $500 \mathrm{~W} 1$ st St, Arlington, TX 76010 \\ ${ }^{c}$ Institute of Mathematics - École Polytechnique Fédérale de Lausanne \\ CH-1015 Lausanne, Switzerland \\ ${ }^{d}$ Instituto di Matematica Applicata e Tecnologie Informatiche "E. Magenes" (CNR) \\ via Ferrata 1, 27100 Pavia, Italy
}

\begin{abstract}
A novel approach which combines isogeometric collocation and an equilibriumbased stress recovery technique is applied to analyze laminated composite plates. Isogeometric collocation is an appealing strong form alternative to standard Galerkin approaches, able to achieve high order convergence rates coupled with a significantly reduced computational cost. Laminated composite plates are herein conveniently modeled considering only one element through the thickness with homogenized material properties. This guarantees accurate results in terms of displacements and in-plane stress components. To recover an accurate out-of-plane stress state, equilibrium is imposed in strong form as a post-processing correction step, which requires the shape functions to be highly continuous. This continuity demand is fully granted
\end{abstract}

\footnotetext{
${ }^{*}$ Corresponding author. E-mail: alessia. patton01@universitadipavia.it
} 
by isogeometric analysis properties, and excellent results are obtained using a minimal number of collocation points per direction, particularly for increasing values of length-to-thickness plate ratio and number of layers. Keywords: Isogeometric Collocation, Splines, Orthotropic materials, Homogenization, Laminated composite plates, Stress recovery procedure

\section{Introduction}

Composite materials consist of two or more materials which combined present enhanced properties that could not be acquired employing any of the constituents alone (see, e.g., [28, 41, 65, 74] and references therein). The interest for composite structures in the engineering community has constantly grown in recent years due to their appealing mechanical properties such as increased stiffness and strength, reduced weight, improved corrosion and wear resistance, just to recall some of them. The majority of man-made composite materials consists of reinforced fibers embedded in a base material, called matrix (see, e.g., [34, 65]). The matrix material keeps the fibers together, acts as a load-transfer medium between fibers, process which takes place through shear stresses, and protects those elements from being exposed to the environment, while the resistence properties of composites are given by the fibers which are stiffer and stronger than the soft matrix. In this paper we focus on laminated composite materials, which are formed by a collection of building blocks or plies, stacked to achieve the desired stiffness and thickness. For this kind of structures also simple loading conditions, such as traction or bending, cause a complex 3D stress state because of the difference in the material properties between the layers, which may lead to delamination, con- 
sequently requiring an accurate stress evaluation through the thickness (see, e.g., [65, 70]). As an alternative to two-dimensional theories, often insufficiently accurate to depict delamination and interlaminar damage, and to layerwise theories, which typically show an high computational cost, a novel method combining an isogeometric analysis (IGA) Galerkin approach with a stress-recovery technique has been recently proposed in [23]. Introduced in 2005 by Hughes et al. [36], IGA aims at integrating design and analysis employing shape functions typically belonging to Computer Aided Design field (such as, B-Splines and NURBS). Using the same shape functions to approximate both geometry and field variables leads to a cost-saving simplification of expensive mesh generation and refinement processes required by standard finite element analyisis. One of the most important features of IGA is the high-regularity of its basis functions leading to superior approximation properties. IGA proved to be successful in a wide variety of problems ranging from solids and structures (see, e.g., [5, 11, 13, 22, 24, 37, 39, 49, 58]) to fluids (see, e.g., [1, 12, 30, 50]), fluid-structure interaction (see, e.g., [10, 35]), opening also the door to geometrically flexible discretizations of higher-order partial differential equations in primal form as in [3, 29, 43, 51]. However, a well-known important issue of IGA is related to the development of efficient integration rules when higher-order approximations are employed (see, e.g., [7, 26, 38, 67]). In attempt to address this problem taking full advantage of the special possibilities offered by IGA, isogeometric collocation (IGA-C) schemes have been proposed in [4]. The aim was to optimize the computational cost still relying on IGA geometrical flexibility and accuracy. Collocation main idea, in contrast to Galerkin-type formulations, consists 
in the discretization of the governing partial differential equations in strong form, evaluated at suitable points. Since integration is not required, isogeometric collocation results in a very fast method providing superior performance in terms of accuracy-to-computational effort ratio with respect to Galerkin formulations, in particular when higher-order approximation degrees are adopted (see [68]). Isogeometric collocation has been particularly successful in the context of structural elements, where isogeometric collocation has proven to be particularly stable in the context of mixed methods. In particular, Bernoulli-Euler beam and Kirchhoff plate elements have been proposed [64], while mixed formulations both for Timoshenko initially-straight planar [17] and non-prismatic [9] beams as well as for curved spatial rods [8] have been introduced and studied, and then effectively extended to the geometrically nonlinear case [47, 53, 54, 55, 75, 77]. Isogeometric collocation has been moreover successfully applied to the solution of Reissner-Mindlin plate problems in [44], and new formulations for shear-deformable beams [45, 47], as well as shells [46, 56] have been solved also via IGA collocation. Since its introduction, many promising significant works on isogeometric collocation methods have been published also in other fields, including phase-field modeling [31], contact [21, 48, 76] and poromechanics [59]. Moreover, combinations with different spline spaces, like hierarchical splines, generalized B-Splines, and T-Splines, have been successfully tested in [14, 52, 68], while alternative effective selection strategies for collocation points have been proposed in [2, 31, 57]. IGA-Galerkin methods have already been used to solve composite laminate problems, especially relying on high-order theories for enhanced plate and shell theories [27, 42, 66, 69, 72]. Recently an isogeometric 
collocation numerical formulation has been proposed [61] to study ReissnerMindlin composite plates. Other Galerkin methods [32, 33, 66] compute instead a full 3D stress state using isogeometric analysis, applying a layerwise technique. This method can also be applied to isogeometric collocation adopting a multipatch approach, which models each layer as a patch (see Figure 1(a) , enforcing normal stress continuity at the inter-patches boundaries [6]. Clearly a layerwise method exploits a number of degrees of freedom directly proportional to the number of layers, inevitably leading to high computational costs. In this paper we apply a single patch 3D isogeometric collocation method to analyze the behavior of composite plates. We adopt a homogenized single-element approach (see Figure 1(b)), which conveniently uses one element through the thickness, coupled with a post-processing technique in order to recover a proper out-of-plane stress state. This method is significantly less expensive compared to a layerwise approach since employs a considerably lower number of degrees of freedom.

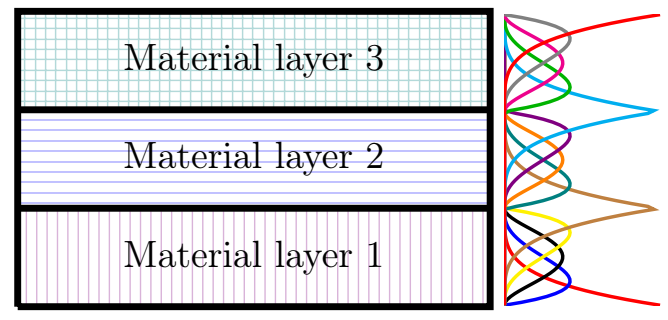

(a) Multipatch approach

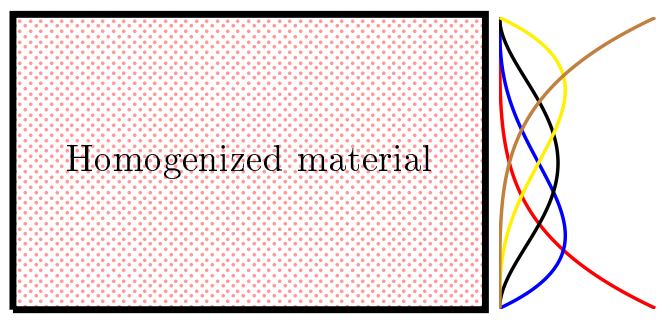

(b) Homogenized single-element approach

Figure 1: Layerwise approach and homogenized single-element example of isogeometric shape functions for a degree of approximation equal to 4 .

The post-processing approach, first proposed in [23], takes inspiration from recovery techniques which can be found in [16, 19, 25, 42, 63, 73] and is based on the direct integration of the equilibrium equations to compute the 
out-of-plane stress components from the in-plane ones directly derived from a coarse displacement solution.

The structure of the paper is organized as follows. In Section 2 the fundamental concepts of multivariate B-Splines and NURBS are presented, followed by an introduction to isogeometric collocation and a description of our IGA-C scheme for orthotropic elasticity. In Section 3 we define our isogeometric collocation strategy to study laminated plates, which combines a homogenized single-element approach with an equilibrium-based stress recovery technique. In Section 4 we present our reference test case and provide results for the single-element approach. Several numerical benchmarks are displayed, which show a significant improvement between non-treated and post-processed outof-plane stress components. Finally we provide some mesh sensitivity tests considering an increasing length-to-thickness ratio and numbers of layers to show the effectiveness of the method. We draw our conclusions in Section 5 .

\section{Isogeometric Collocation: Basics and application to orthotropic elasticity}

In this section we introduce the notions of multivariate B-Splines and NURBS, provide some details regarding isogeometric collocation and describe our collocation scheme in the context of linear orthotropic elasticity.

\subsection{Multivariate B-Splines and NURBS}

In the following, we introduce the basic definitions and notations about multivariate B-Splines and NURBS. For further details, readers may refer to [15, 36, 62], and references therein. Multivariate B-Splines are generated through the tensor product of univariate B-Splines. We denote with $d_{p}$ the 
dimension of the parametric space and therefore $d_{p}$ univariate knot vectors have to be introduced as

$$
\Theta=\left\{\theta_{1}^{d}, \ldots, \theta_{m_{d}+p_{d}+1}^{d}\right\} \quad d=1, \ldots, d_{p}
$$

where $p_{d}$ represents the polynomial degree in the parametric direction $d$, and $m_{d}$ is the associated number of basis functions. Given the univariate basis functions $N_{i_{d}, p_{d}}^{d}$ associated to each parametric direction $\xi^{d}$, the multivariate basis functions $B_{\mathbf{i}, \mathbf{p}}(\boldsymbol{\xi})$ are obtained as:

$$
B_{\mathbf{i}, \mathbf{p}}(\boldsymbol{\xi})=\prod_{d=1}^{d_{p}} N_{i_{d}, p_{d}}\left(\xi^{d}\right)
$$

where $\mathbf{i}=\left\{i_{1}, \ldots, i_{d_{p}}\right\}$ plays the role of a multi-index which describes the considered position in the tensor product structure, $\mathbf{p}=\left\{p_{1}, \ldots, p_{d}\right\}$ indicates the polynomial degrees, and $\boldsymbol{\xi}=\left\{\xi^{1}, \ldots, \xi^{d_{p}}\right\}$ represents the vector of the parametric coordinates in each parametric direction $d$. B-Spline multidimensional geometries are built from a linear combination of multivariate B-Spline basis functions as follows

$$
\mathbf{S}(\boldsymbol{\xi})=\sum_{\mathbf{i}} B_{\mathbf{i}, \mathbf{p}}(\boldsymbol{\xi}) \mathbf{P}_{\mathbf{i}}
$$

where the coefficients $\mathbf{P}_{\mathbf{i}} \in \mathbb{R}^{d_{s}}$ of the linear combination are the so-called control points ( $d_{s}$ is the dimension of the physical space) and the summation is extended to all combinations of the multi-index $\mathbf{i}$. NURBS geometries in $\mathbb{R}^{d_{s}}$ are instead obtained from a projective transformation of their B-Spline counterparts in $\mathbb{R}^{d_{s}+1}$. Defining $w_{\mathbf{i}}$ as the collection of weights according to the multi-index i, multivariate NURBS basis functions are obtained as

$$
R_{\mathbf{i}, \mathbf{p}}(\boldsymbol{\xi})=\frac{B_{\mathbf{i}, \mathbf{p}}(\boldsymbol{\xi}) w_{\mathbf{i}}}{\sum_{\mathbf{j}} B_{\mathbf{j}, \mathbf{p}}(\boldsymbol{\xi}) w_{\mathbf{j}}}
$$


and NURBS multidimensional geometries are built as

$$
\mathbf{S}(\boldsymbol{\xi})=\sum_{\mathbf{i}} R_{\mathbf{i}, \mathbf{p}}(\boldsymbol{\xi}) \mathbf{P}_{\mathbf{i}}
$$

\subsection{An Introduction to Isogeometric collocation}

Collocation methods have been introduced within isogeometric analysis as an attempt to address a well-known important issue of early IGA-Galerkin formulations, related to the development of efficient integration rules for higher-order approximations. In fact, element-wise Gauss quadrature, typically used for finite elements and originally adopted for Galerkin-based IGA, does not properly take into account inter-element higher continuity leading to sub-optimal array formation and assembly costs, significantly affecting the performance of IGA methods. Isogeometric collocation aimed at optimizing computational cost, since it may be viewed as a variant of one-point quadrature numerical scheme, still taking advantage of IGA geometrical flexibility and accuracy. Collocation methods are based on the direct discretization in strong form of the differential equations governing the problem evaluated at suitable points. The isoparametric paradigm is adopted and the same basis functions are used to describe both geometry and problem unknowns. Once the approximations are carried out, as in a typical Galerkin-IGA context, by means of a linear combinations of IGA basis functions and control variables, the discrete differential equations are collocated at each collocation point. Consequently a delicate issue is represented by the determination of suitable collocation points. A widespread approach which is proposed in the engineering literature is to collocate at the images of Greville abscissae (see, e.g., [40]), but this represents just the simplest possible option (see, 
e.g., [18, 20] for alternative choices). Along each parametric direction $d$, Greville abscissae consist of a set of $m^{d}$ points, obtained from the knot vector components, $\theta_{i}^{d}$, as

$$
\bar{\theta}_{i}^{d}=\frac{\theta_{i+1}^{d}+\theta_{i+2}^{d}+\ldots+\theta_{i+p}^{d}}{p_{d}} \quad i=1, \ldots, m^{d}
$$

$p_{d}$ being the degree of approximation. Since the approximation is performed through direct collocation of the differential equations, no integrals need to be computed and consequently, evaluation and assembly operations lead to a significantly reduced computational cost.

\subsection{Numerical formulation for orthotropic elasticity}

Once a strategy to select collocation points and compute IGA shape functions is set, a proper description of the equations in strong form for the problem under examination is required, as mentioned in Section 2.2. We therefore recall the classical elasticity problem in strong form considering a small strain regime and detail equilibrium equations using Einstein's notation (7). The following notations are used: $\Omega \subset \mathbb{R}^{3}$, is an open bounded domain, representing an elastic three-dimensional body, $\Gamma_{N}$ and $\Gamma_{D}$ are defined as boundary portions subjected respectively to Neumann and Dirichlet conditions such that $\Gamma_{N} \cup \Gamma_{D}=\partial \Omega$ and $\Gamma_{N} \cap \Gamma_{D}=\varnothing$. Accordingly, the equilibrium equations and the corresponding boundary conditions are:

$$
\begin{array}{ll}
\sigma_{i j, j}+b_{i}=0 & \text { in } \Omega \\
\sigma_{i j} n_{j}=t_{i} & \text { on } \Gamma_{N} \\
u_{i}=\bar{u}_{i} & \text { on } \Gamma_{D}
\end{array}
$$


where $\sigma_{i j}$ and $u_{i}$ represent respectively the Cauchy stress and displacement components, while $b_{i}$ and $t_{i}$ the volume and traction forces, $n_{j}$ the outward normal, and $\bar{u}_{i}$ the prescribed displacements. The elasticity problem is finally completed by the kinematic relations in small strain

$$
\varepsilon_{i j}=\frac{u_{i, j}+u_{j, i}}{2}
$$

as well as by the constitutive equations

$$
\sigma_{i j}=\mathbb{C}_{i j k m} \varepsilon_{k m}
$$

where $\mathbb{C}_{i j k m}$ is the fourth order elasicity tensor.

As we described in Section 1, the basic building block of a laminate is a lamina, i.e., a flat arrangement of unidirectional fibers, considering the simplest case, embedded in a matrix. In order to increase the composite resistance properties cross-ply laminates can be employed (i.e., all the plies used to form the composite stacking sequence are piled alternating different fiber layers orientations) in which all unidirectional layers are individually orthotropic. Since the proposed collocation approach uses one element through the thickness to model the composite plate as a homogenized single building block, we focus in this section on the collocation formulation for a plate formed by only one orthotropic elastic lamina. Considering three mutually orthogonal planes of material symmetry for each ply, the number of elastic coefficients of the fourth order elasticity tensor $\mathbb{C}_{i j k m}$ is reduced to 9 in Voigt notation, that can be expressed in terms of engineering constants as 


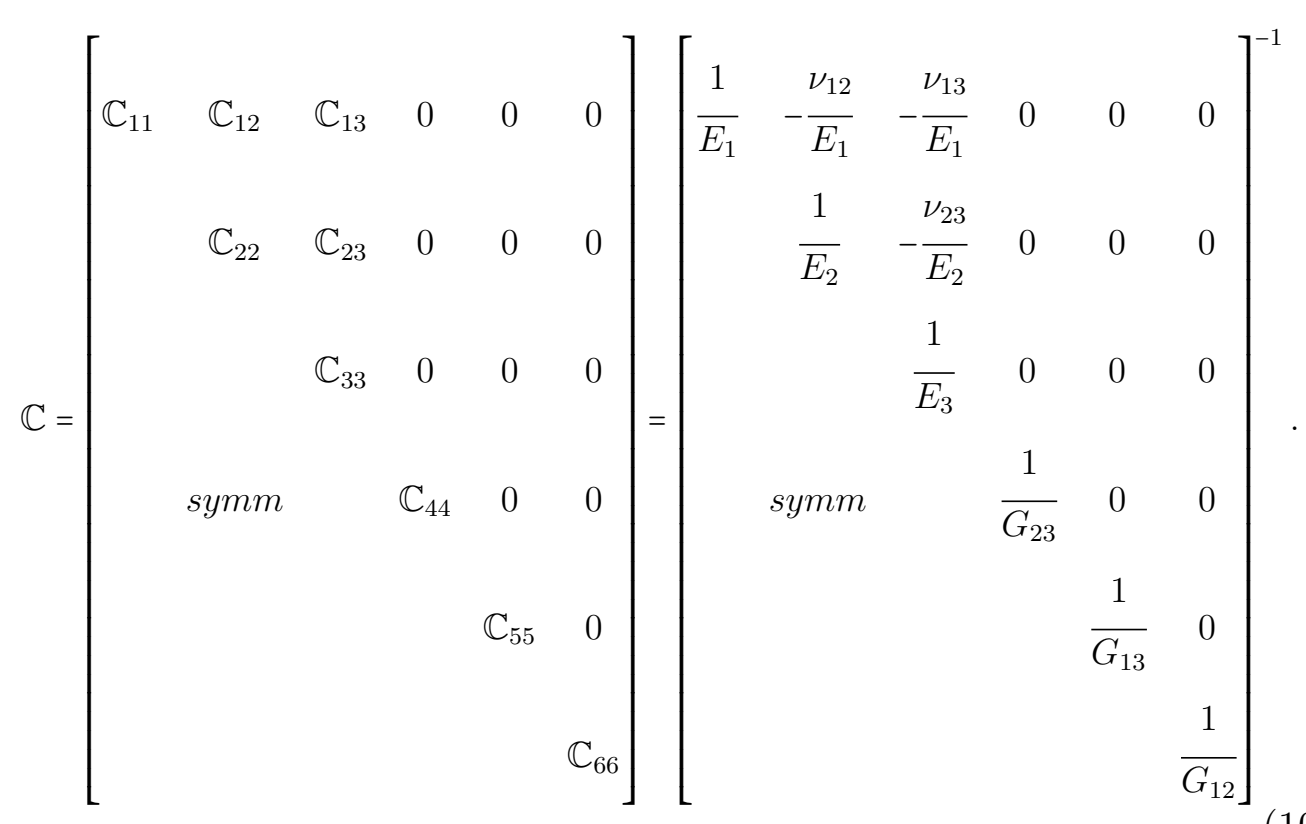

The displacement field is then approximate as a linear combination of NURBS multivariate shape functions and control points as follows

$$
\begin{aligned}
& \mathbf{u}(\boldsymbol{\xi})=R_{\mathbf{i}, \mathbf{p}}(\boldsymbol{\xi}) \hat{\mathbf{u}}_{\mathbf{i}}, \\
& \mathbf{v}(\boldsymbol{\xi})=R_{\mathbf{i}, \mathbf{p}}(\boldsymbol{\xi}) \hat{\mathbf{v}}_{\mathbf{i}}, \\
& \mathbf{w}(\boldsymbol{\xi})=R_{\mathbf{i}, \mathbf{p}}(\boldsymbol{\xi}) \hat{\mathbf{w}}_{\mathbf{i}} .
\end{aligned}
$$

Having defined $\boldsymbol{\tau}$ as the matrix of collocation points, we insert the approximations (11) into kinematics equations (8) and we combine the obtained expressions with the constitutive relations (9). Finally we substitute into equilibrium equations $7 \mathrm{a}$ obtaining

$$
\left[\begin{array}{ccc}
\mathbf{K}_{11}(\boldsymbol{\tau}) & \mathbf{K}_{12}(\boldsymbol{\tau}) & \mathbf{K}_{13}(\boldsymbol{\tau}) \\
& \mathbf{K}_{22}(\boldsymbol{\tau}) & \mathbf{K}_{23}(\boldsymbol{\tau}) \\
\text { symm } & & \mathbf{K}_{33}(\boldsymbol{\tau})
\end{array}\right] \cdot\left(\begin{array}{c}
\hat{\mathbf{u}}_{\mathbf{i}} \\
\hat{\mathbf{v}}_{\mathbf{i}} \\
\hat{\mathbf{w}}_{\mathbf{i}}
\end{array}\right)=-\mathbf{b}(\boldsymbol{\tau}), \quad \forall \boldsymbol{\tau} \in \Omega
$$


where $\mathbf{K}_{i j}(\boldsymbol{\tau})$ cofficients can be expressed as

$$
\begin{aligned}
& \mathbf{K}_{11}(\boldsymbol{\tau})=\mathbb{C}_{11} \frac{\partial^{2} R_{\mathbf{i}, \mathbf{p}}(\boldsymbol{\tau})}{\partial x_{1}{ }^{2}}+\mathbb{C}_{66} \frac{\partial^{2} R_{\mathbf{i}, \mathbf{p}}(\boldsymbol{\tau})}{\partial x_{2}{ }^{2}}+\mathbb{C}_{55} \frac{\partial^{2} R_{\mathbf{i}, \mathbf{p}}(\boldsymbol{\tau})}{\partial x_{3}{ }^{2}} \\
& \mathbf{K}_{22}(\boldsymbol{\tau})=\mathbb{C}_{66} \frac{\partial^{2} R_{\mathbf{i}, \mathbf{p}}(\boldsymbol{\tau})}{\partial x_{1}^{2}}+\mathbb{C}_{22} \frac{\partial^{2} R_{\mathbf{i}, \mathbf{p}}(\boldsymbol{\tau})}{\partial x_{2}{ }^{2}}+\mathbb{C}_{44} \frac{\partial^{2} R_{\mathbf{i}, \mathbf{p}}(\boldsymbol{\tau})}{\partial x_{3}{ }^{2}} \\
& \mathbf{K}_{33}(\boldsymbol{\tau})=\mathbb{C}_{55} \frac{\partial^{2} R_{\mathbf{i}, \mathbf{p}}(\boldsymbol{\tau})}{\partial x_{1}{ }^{2}}+\mathbb{C}_{44} \frac{\partial^{2} R_{\mathbf{i}, \mathbf{p}}(\boldsymbol{\tau})}{\partial x_{2}{ }^{2}}+\mathbb{C}_{33} \frac{\partial^{2} R_{\mathbf{i}, \mathbf{p}}(\boldsymbol{\tau})}{\partial x_{3}{ }^{2}} \\
& \mathbf{K}_{23}(\boldsymbol{\tau})=\left(\mathbb{C}_{23}+\mathbb{C}_{44}\right) \frac{\partial^{2} R_{\mathbf{i}, \mathbf{p}}(\boldsymbol{\tau})}{\partial x_{2} \partial x_{3}} \\
& \mathbf{K}_{13}(\boldsymbol{\tau})=\left(\mathbb{C}_{13}+\mathbb{C}_{55}\right) \frac{\partial^{2} R_{\mathbf{i}, \mathbf{p}}(\boldsymbol{\tau})}{\partial x_{1} \partial x_{3}} \\
& \mathbf{K}_{12}(\boldsymbol{\tau})=\left(\mathbb{C}_{12}+\mathbb{C}_{66}\right) \frac{\partial^{2} R_{\mathbf{i}, \mathbf{p}}(\boldsymbol{\tau})}{\partial x_{1} \partial x_{2}}
\end{aligned}
$$

and substituting in $7 \mathrm{~b}$ we obtain:

$$
\left[\begin{array}{ccc}
\tilde{\mathbf{K}}_{11}(\boldsymbol{\tau}) & \tilde{\mathbf{K}}_{12}(\boldsymbol{\tau}) & \tilde{\mathbf{K}}_{13}(\boldsymbol{\tau}) \\
& \tilde{\mathbf{K}}_{22}(\boldsymbol{\tau}) & \tilde{\mathbf{K}}_{23}(\boldsymbol{\tau}) \\
\text { symm } & & \tilde{\mathbf{K}}_{33}(\boldsymbol{\tau})
\end{array}\right] \cdot\left(\begin{array}{c}
\hat{\mathbf{u}}_{\mathbf{i}} \\
\hat{\mathbf{v}}_{\mathbf{i}} \\
\hat{\mathbf{w}}_{\mathbf{i}}
\end{array}\right)=\mathbf{t}(\boldsymbol{\tau}), \quad \forall \boldsymbol{\tau} \in \Gamma_{N}
$$

with $\tilde{\mathbf{K}}_{i j}(\boldsymbol{\tau})$ components having the following form

$$
\begin{aligned}
& \tilde{\mathbf{K}}_{11}(\boldsymbol{\tau})=\mathbb{C}_{11} \frac{\partial R_{\mathbf{i}, \mathbf{p}}(\boldsymbol{\tau})}{\partial x_{1}} n_{1}+\mathbb{C}_{66} \frac{\partial R_{\mathbf{i}, \mathbf{p}}(\boldsymbol{\tau})}{\partial x_{2}} n_{2}+\mathbb{C}_{55} \frac{\partial R_{\mathbf{i}, \mathbf{p}}(\boldsymbol{\tau})}{\partial x_{3}} n_{3}, \\
& \tilde{\mathbf{K}}_{22}(\boldsymbol{\tau})=\mathbb{C}_{66} \frac{\partial R_{\mathbf{i}, \mathbf{p}}(\boldsymbol{\tau})}{\partial x_{1}} n_{1}+\mathbb{C}_{22} \frac{\partial R_{\mathbf{i}, \mathbf{p}}(\boldsymbol{\tau})}{\partial x_{2}} n_{2}+\mathbb{C}_{44} \frac{\partial R_{\mathbf{i}, \mathbf{p}}(\boldsymbol{\tau})}{\partial x_{3}} n_{3}, \\
& \tilde{\mathbf{K}}_{33}(\boldsymbol{\tau})=\mathbb{C}_{55} \frac{\partial R_{\mathbf{i}, \mathbf{p}}(\boldsymbol{\tau})}{\partial x_{1}} n_{1}+\mathbb{C}_{44} \frac{\partial R_{\mathbf{i}, \mathbf{p}}(\boldsymbol{\tau})}{\partial x_{2}} n_{2}+\mathbb{C}_{33} \frac{\partial R_{\mathbf{i}, \mathbf{p}}(\boldsymbol{\tau})}{\partial x_{3}} n_{3}, \\
& \tilde{\mathbf{K}}_{23}(\boldsymbol{\tau})=\mathbb{C}_{23} \frac{\partial R_{\mathbf{i}, \mathbf{p}}(\boldsymbol{\tau})}{\partial x_{3}} n_{2}+\mathbb{C}_{44} \frac{\partial R_{\mathbf{i}, \mathbf{p}}(\boldsymbol{\tau})}{\partial x_{2}} n_{3},
\end{aligned}
$$




$$
\begin{aligned}
\tilde{\mathbf{K}}_{13}(\boldsymbol{\tau}) & =\mathbb{C}_{13} \frac{\partial R_{\mathbf{i}, \mathbf{p}}(\boldsymbol{\tau})}{\partial x_{3}} n_{1}+\mathbb{C}_{55} \frac{\partial R_{\mathbf{i}, \mathbf{p}}(\boldsymbol{\tau})}{\partial x_{1}} n_{3}, \\
\tilde{\mathbf{K}}_{12}(\boldsymbol{\tau}) & =\mathbb{C}_{12} \frac{\partial R_{\mathbf{i}, \mathbf{p}}(\boldsymbol{\tau})}{\partial x_{2}} n_{1}+\mathbb{C}_{66} \frac{\partial R_{\mathbf{i}, \mathbf{p}}(\boldsymbol{\tau})}{\partial x_{1}} n_{2} .
\end{aligned}
$$

As we can see from equations (13), Neumann boundary conditions are directly imposed as strong equations at the collocation points belonging to the boundary surface (see, [6, 21]), with the usual physical meaning of prescribed boundary traction.

\section{An IGA collocation approach to model $3 \mathrm{D}$ composite plates}

In this section we describe our IGA 3D collocation strategy to model composite plates. The proposed method, known as single element approach, relies on a homogenization technique combined with a post-processing approach based on the imposition of equilibrium equations in strong form.

\subsection{Single-element approach}

The single-element approach considers the plate discretized by a single element through the thickness, which strongly reduces the number of degrees of freedom with respect to layerwise methods. The material matrix is therefore homogenized to account for the presence of the layers as Figure 1(b) clearly describes.

Remark 1. Considering a single-element homogenized approach is effective only for through-the-thickness symmetric layer distributions, as for nonsymmetric ply stacking sequences the plate middle plane is not balanced. In the case of non-symmetric layer distributions this technique is still applicable when the stacking sequence can be split into two symmetric piles, using one element per homogenized stack with a $C^{0}$ interface. 
This method provides accurate results only in terms of displacements and in-plane stress components and, in order to recover a proper out-of-plane stress state, following [23], we propose to couple it with a post-processing technique. To characterize the variation of the material properties from layer to layer, we homogenize the constitutive behavior to create an equivalent single-layer laminate, referring to [71], where explicit expressions for the effective elastic constants of the equivalent laminate are given as

$$
\begin{aligned}
& \overline{\mathbb{C}}_{11}=\sum_{k=1}^{N} \bar{t}_{k} \mathbb{C}_{11}^{(k)}+\sum_{k=2}^{N}\left(\mathbb{C}_{13}^{(k)}-\overline{\mathbb{C}}_{13}\right) \bar{t}_{k} \frac{\left(\mathbb{C}_{13}^{(1)}-\mathbb{C}_{13}^{(k)}\right)}{\mathbb{C}_{33}^{(k)}} \\
& \overline{\mathbb{C}}_{12}=\sum_{k=1}^{N} \bar{t}_{k} \mathbb{C}_{12}^{(k)}+\sum_{k=2}^{N}\left(\mathbb{C}_{13}^{(k)}-\overline{\mathbb{C}}_{13}\right) \bar{t}_{k} \frac{\left(\mathbb{C}_{23}^{(1)}-\mathbb{C}_{23}^{(k)}\right)}{\mathbb{C}_{33}^{(k)}} \\
& \overline{\mathbb{C}}_{13}=\sum_{k=1}^{N} \bar{t}_{k} \mathbb{C}_{13}^{(k)}+\sum_{k=2}^{N}\left(\mathbb{C}_{33}^{(k)}-\overline{\mathbb{C}}_{33}\right) \bar{t}_{k} \frac{\left(\mathbb{C}_{13}^{(1)}-\mathbb{C}_{13}^{(k)}\right)}{\mathbb{C}_{33}^{(k)}} \\
& \overline{\mathbb{C}}_{22}=\sum_{k=1}^{N} \bar{t}_{k} \mathbb{C}_{22}^{(k)}+\sum_{k=2}^{N}\left(\mathbb{C}_{23}^{(k)}-\overline{\mathbb{C}}_{23}\right) \bar{t}_{k} \frac{\left(\mathbb{C}_{23}^{(1)}-\mathbb{C}_{23}^{(k)}\right)}{\mathbb{C}_{33}^{(k)}} \\
& \overline{\mathbb{C}}_{23}=\sum_{k=1}^{N} \bar{t}_{k} \mathbb{C}_{23}^{(k)}+\sum_{k=2}^{N}\left(\mathbb{C}_{33}^{(k)}-\overline{\mathbb{C}}_{33}\right) \bar{t}_{k} \frac{\left(\mathbb{C}_{23}^{(1)}-\mathbb{C}_{23}^{(k)}\right)}{\mathbb{C}_{33}^{(k)}} \\
& \overline{\mathbb{C}}_{33}=\frac{1}{\left(t_{k}\right)} \\
& \left(\sum_{k=1}^{N} \frac{\bar{t}_{k}}{\mathbb{C}_{33}^{(k)}}\right) \\
& \overline{\mathbb{C}}_{44}=\frac{\left(\sum_{k=1}^{N} \frac{\bar{t}_{k} \mathbb{C}_{44}^{(k)}}{\Delta_{k}}\right)}{\Delta}, \quad \Delta=\left(\sum_{k=1}^{N} \frac{\bar{t}_{k} \mathbb{C}_{44}^{(k)}}{\Delta_{k}}\right)\left(\sum_{k=1}^{N} \frac{\bar{t}_{k} \mathbb{C}_{55}^{(k)}}{\Delta_{k}}\right) \\
& \overline{\mathbb{C}}_{55}=\frac{\left(\sum_{k=1}^{N} \frac{\bar{t}_{k} \mathbb{C}_{55}^{(k)}}{\Delta_{k}}\right)}{\Delta}, \quad \Delta_{k}=\mathbb{C}_{44}^{k} \mathbb{C}_{55}^{k} \\
& \overline{\mathbb{C}}_{66}=\sum_{k=1}^{N} \bar{t}_{k} \mathbb{C}_{66}^{(k)}
\end{aligned}
$$


where $\mathbb{C}_{i j}^{(k)}$ represents the $i j$-th component of the fourth order elasticity tensor in Voigt notation (10) for the $k$-th layer and $\bar{t}_{k}=\frac{t_{k}}{h}$ stands for the volume fraction of the $k$-th lamina, $h$ being the total thickness and $t_{k}$ the $k$-th thickness.

\subsubsection{Post-processing step: Reconstruction from Equilibrium}

As interlaminar delamination and other fracture processes rely mostly on out-of-plane components, a proper through-the-thickness stress description is required. In order to recover a more accurate stress state, we perform a postprocessing step based on the equilibrium equations, following [23], relying on the higher regularity granted by IGA shape functions. This procedure, which takes its roots in [19, 25, 63, 73], has already been proven to be successful for IGA-Galerkin. Inside the plate the stresses should satisfy the equilibrium equation 7 a that can be expanded as

$$
\begin{aligned}
& \sigma_{11,1}+\sigma_{12,2}+\sigma_{13,3}=-b_{1}, \\
& \sigma_{12,1}+\sigma_{22,2}+\sigma_{23,3}=-b_{2} \\
& \sigma_{13,1}+\sigma_{23,2}+\sigma_{33,3}=-b_{3} .
\end{aligned}
$$

Assuming the in-plane stress components to well approximate the laminate behaviour, as it will be shown in Section 4 , we can integrate equation $15 \mathrm{a}$ and $15 \mathrm{~b}$ along the thickness, recovering the out-of-plane shear stresses as

$$
\begin{aligned}
& \sigma_{13}\left(X_{3}\right)=-\int_{\bar{X}_{3}}^{X_{3}}\left(\sigma_{11,1}(\zeta)+\sigma_{12,2}(\zeta)+b_{1}(\zeta)\right) \mathrm{d} \zeta+\sigma_{13}\left(\bar{X}_{3}\right) \\
& \sigma_{23}\left(X_{3}\right)=-\int_{\bar{X}_{3}}^{X_{3}}\left(\sigma_{12,1}(\zeta)+\sigma_{22,2}(\zeta)+b_{2}(\zeta)\right) \mathrm{d} \zeta+\sigma_{23}\left(\bar{X}_{3}\right)
\end{aligned}
$$

where $\zeta$ represents the coordinate along the thickness direction.

Finally we can insert equations $16 \mathrm{a})$ and $16 \mathrm{~b}$ into $15 \mathrm{c})$, recovering the $\sigma_{33}$ 
component as

$$
\sigma_{33}\left(X_{3}\right)=-\int_{\bar{X}_{3}}^{X_{3}}\left(\sigma_{13,1}(\zeta)+\sigma_{23,2}(\zeta)+b_{3}(\zeta)\right) \mathrm{d} \zeta+\sigma_{33}\left(\bar{X}_{3}\right)
$$

Following [23], the integral constants are chosen to fulfil the boundary conditions at the top or bottom surfaces.

Recalling that

$$
\sigma_{i j, k}=\overline{\mathbb{C}}_{i j m n} \frac{u_{m, n k}+u_{n, m k}}{2},
$$

where the homogenized elasticity tensor $\overline{\mathbb{C}}$ is constant, it is clear the necessity of a highly regular displacement solution in order to recover a proper stress state. Such a condition can be easily achieved using isogeometric collocation, due to the possibility to benefit from the high regularity of B-Splines or NURBS. We also remark that the proposed method strongly relies on the possibility to obtain an accurate description (with a relatively coarse mesh) of the in-plane stress state.

\section{Numerical tests}

In this section, to assess whether the proposed method can effectively reproduce composite plates behaviour, we consider a classical benchmark problem [60] and we address different aspects such as the effectiveness of the proposed post-processing step, the method sensitivity to parameters of interest (i.e., number of layers and length-to-thickness ratio), and its convergence.

\subsection{Reference solution: the Pagano layered plate}

A square laminated composite plate of total thickness $t$ made of $N$ orthotropic layers is considered. This structure is simply supported and a normal sinusoidal traction is applied on the upper surface, while the lower 


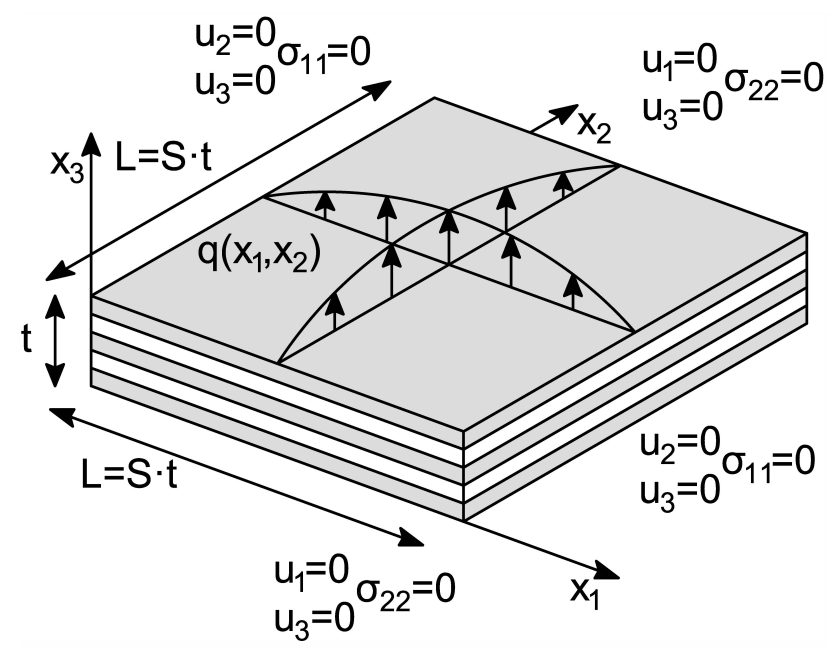

Figure 2: Pagano's test case [60]. Problem geometry and boundary conditions.

surface is traction-free, as shown in Figure 2, In the proposed numerical tests we consider different numbers of layers, namely 3, 11 and 33. The thickness of every single layer is set to $1 \mathrm{~mm}$, and the edge length, $L$, is chosen to be $S$ times larger than the total thickness $t$ of the laminate. Different choices of length-to-thickness ratio are considered (i.e., 20, 30, 40, and 50) which allow to draw interesting considerations about the laminate behaviour in the proposed convergence tests. For all examples we consider the same loading conditions proposed by Pagano, i.e., a double sinus with periodicity equal to twice the length of the plate. As depicted in Figure 2 the laminated plate is composed of layers organized in an alternated distribution of orthotropic plies (i.e., a $0^{\circ} / 90^{\circ}$ stacking sequence in our case). Layer material parameters considered in the numerical tests are summarized in Table 1 for $0^{\circ}$-oriented plies. 
Table 1: Material properties for $0^{\circ}$-oriented layers employed in the numerical tests.

\begin{tabular}{|ccccccccc|}
\hline$E_{1}$ & $E_{2}$ & $E_{3}$ & $G_{23}$ & $G_{13}$ & $G_{12}$ & $\nu_{23}$ & $\nu_{13}$ & $\nu_{12}$ \\
\hline$[\mathrm{GPa}]$ & {$[\mathrm{GPa}]$} & {$[\mathrm{GPa}]$} & {$[\mathrm{GPa}]$} & {$[\mathrm{GPa}]$} & {$[\mathrm{GPa}]$} & {$[-]$} & {$[-]$} & {$[-]$} \\
\hline 25000 & 1000 & 1000 & 200 & 500 & 500 & 0.25 & 0.25 & 0.25 \\
\hline
\end{tabular}

The Neumann boundary conditions on the plate surfaces $x_{3}= \pm \frac{t}{2}$ are

$$
\begin{aligned}
& \sigma_{33}\left(x_{1}, x_{2},-\frac{t}{2}\right)=\sigma_{13}\left(x_{1}, x_{2}, \pm \frac{t}{2}\right)=\sigma_{23}\left(x_{1}, x_{2}, \pm \frac{t}{2}\right)=0, \\
& \sigma_{33}\left(x_{1}, x_{2}, \frac{t}{2}\right)=\sigma_{0} \sin \left(\frac{\pi x_{1}}{S t}\right) \sin \left(\frac{\pi x_{2}}{S t}\right),
\end{aligned}
$$

where $\sigma_{0}=1 \mathrm{MPa}$.

The simple support edge conditions are taken as

- $\sigma_{11}=0$ and $u_{2}=u_{3}=0$ at $x_{1}=0$ and $x_{1}=L$,

- $\sigma_{22}=0$ and $u_{1}=u_{3}=0$ at $x_{2}=0$ and $x_{2}=L$.

All results are then expressed in terms of the following normalized stress components

$$
\begin{array}{ll}
\bar{\sigma}_{i j}=\frac{\sigma_{i j}}{\sigma_{0} S^{2}}, & i, j=1,2, \\
\bar{\sigma}_{i 3}=\frac{\sigma_{i 3}}{\sigma_{0} S}, & i=1,2, \\
\bar{\sigma}_{33}=\frac{\sigma_{33}}{\sigma_{0}} . &
\end{array}
$$

\subsection{Post-processed out-of-plane stresses}

In this section, we comment the results obtained using the proposed IGAcollocation approach, as compared with Pagano's analytical solution [60]. To give an idea of the improvement granted by the post-processing of out-ofplane stress components, in Figures 3 and 4 we compare the reference solution 
with non-treated and post-processed results for the cases with 3 and 11 layers, considering a length to thickness ratio $S=20$. All numerical simulations are carried out using an in-plane degree of approximation $p=q=6$ and 10 collocation points for each in-plane parametric direction, while we use an approximation degree $r=4$ and one element through the thickness (i.e., $r+1$ collocation points). The sampling point where we show results is located at $x_{1}=x_{2}=0.25 L$. For both considered cases the in-plane stresses show a good behaviour, as expected, while the out-of-plane stress components, without a post-processing treatment, are erroneusly discontinuous. The proposed results clearly show the improvement granted by the post-process of out-ofplane components. 


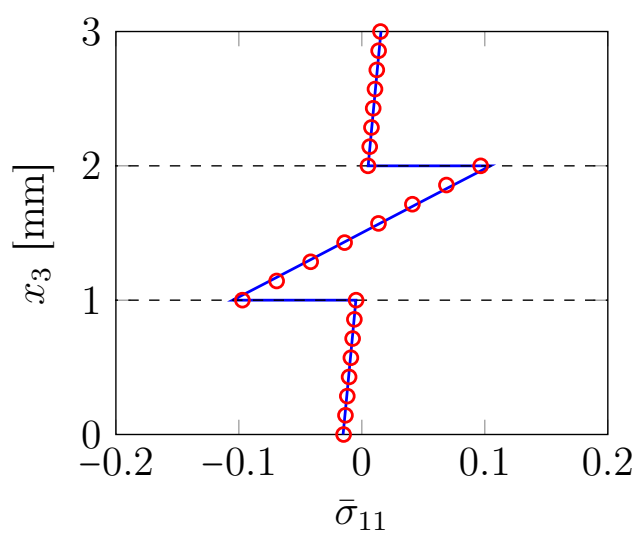

(a) Normalized $\sigma_{11}$

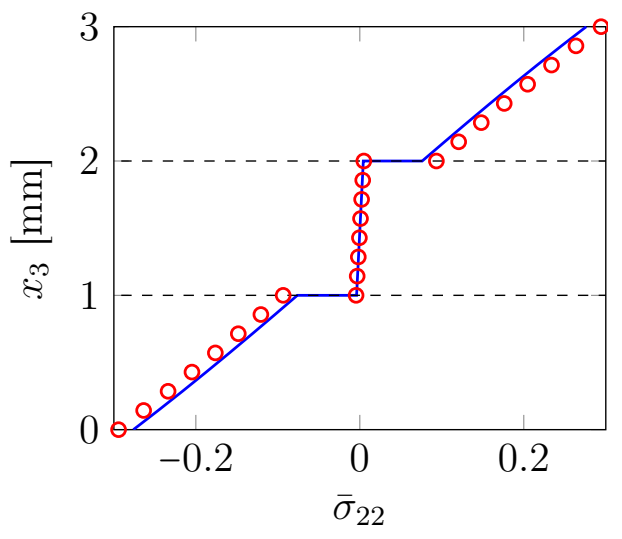

(c) Normalized $\sigma_{22}$

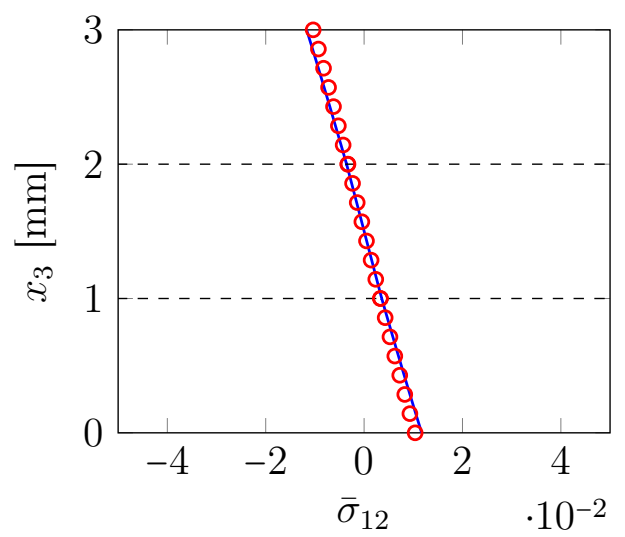

(e) Normalized $\sigma_{12}$

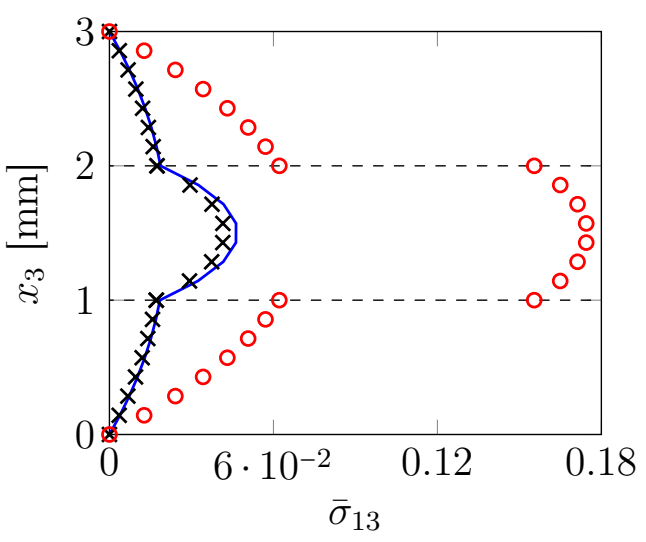

(b) Normalized $\sigma_{13}$

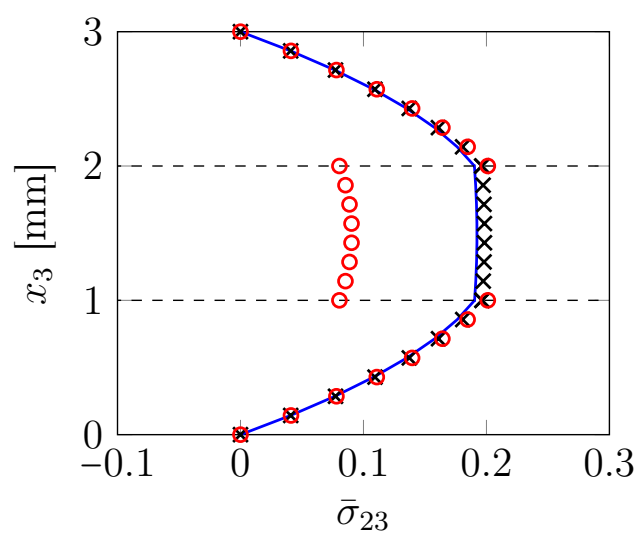

(d) Normalized $\sigma_{23}$

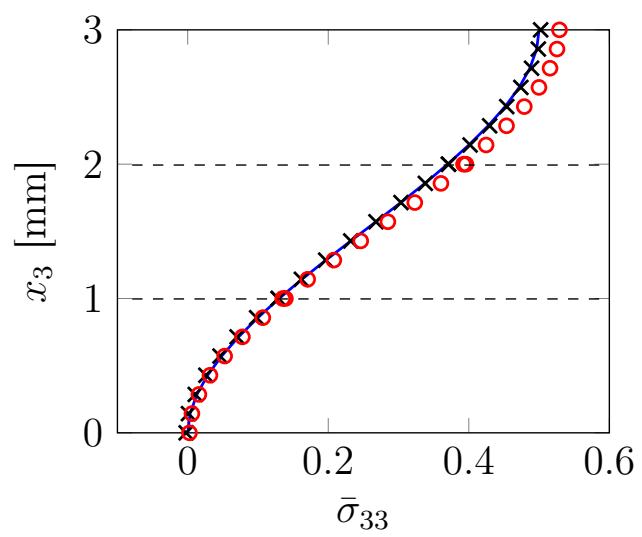

(f) Normalized $\sigma_{33}$

Figure 3: Through-the-thickness stress solutions for the 3D Pagano problem 60 evaluated at $x_{1}=x_{2}=0.25 \mathrm{~L}$. Case: plate with 3 layers and length-to-thickness ratio $S=20$ (- Pagano's solution, o homogenized single-element approach solution (without postprocessing), $\times$ post-processed solution). 


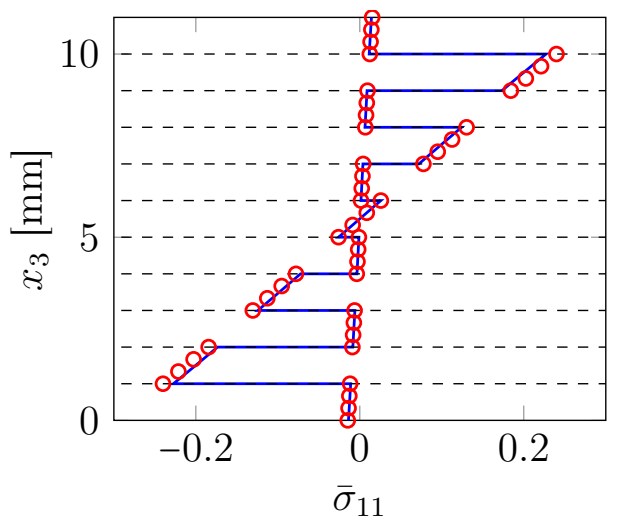

(a) Normalized $\sigma_{11}$

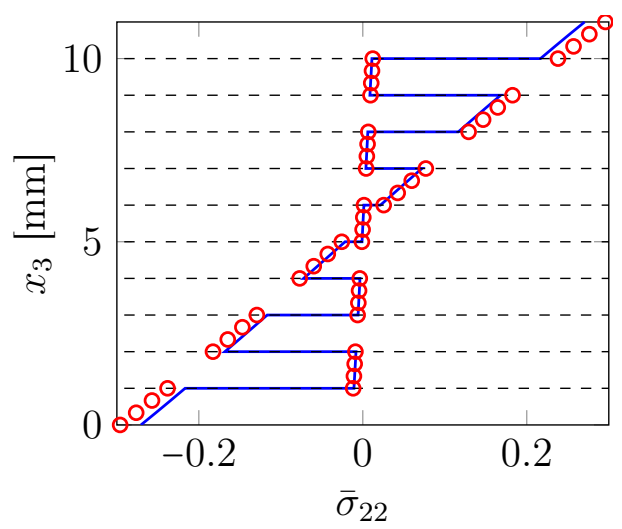

(c) Normalized $\sigma_{22}$

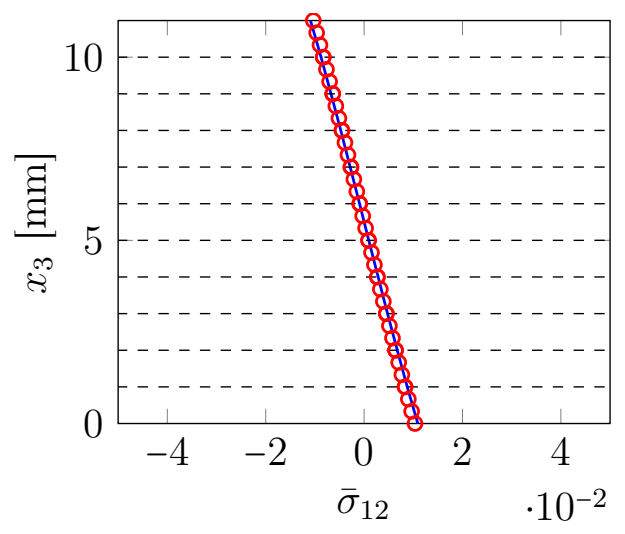

(e) Normalized $\sigma_{12}$

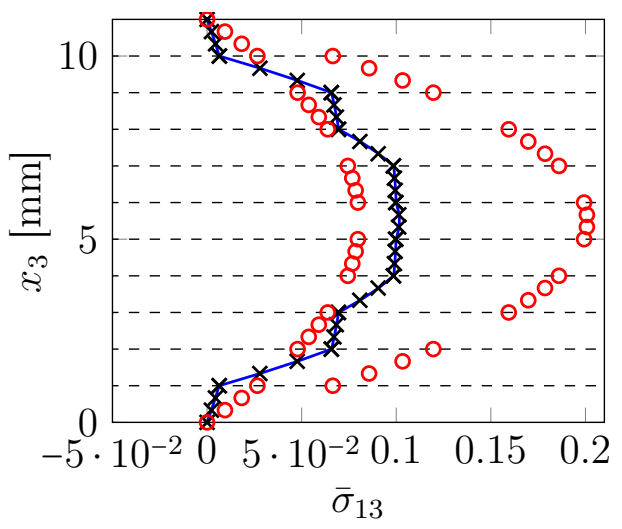

(b) Normalized $\sigma_{13}$

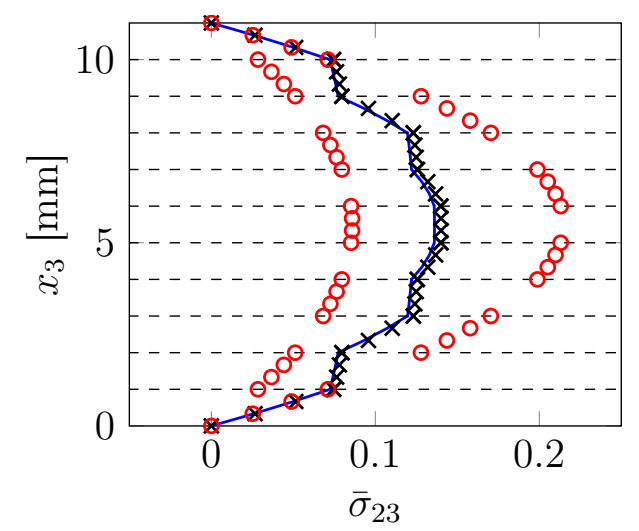

(d) Normalized $\sigma_{23}$

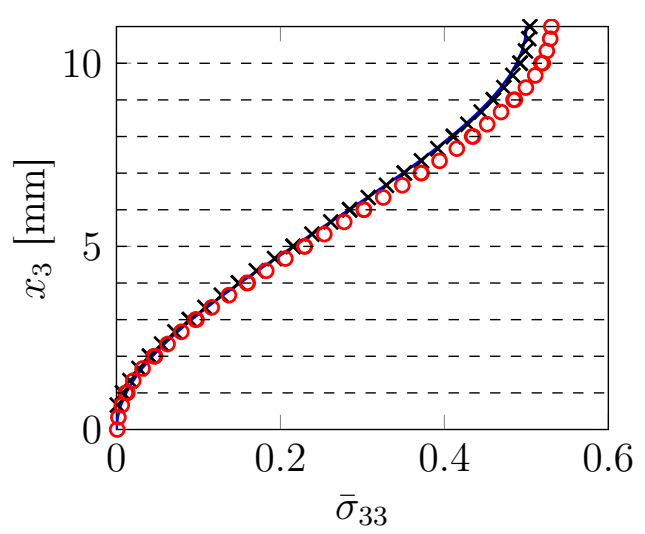

(f) Normalized $\sigma_{33}$

Figure 4: Through-the-thickness stress solutions for the 3D Pagano problem [60] evaluated at $x_{1}=x_{2}=0.25 \mathrm{~L}$. Case: plate with 11 layers and length-to-thickness ratio $S=20$ (- Pagano's solution, o homogenized single-element approach solution (without postprocessing), $\times$ post-processed solution). 
To show the effect of post-processing at different locations of the plate, in Figures 547 the out-of-plane stress state profile is recovered sampling the laminae every quarter of length in both in-plane directions, for the case of a length-to-thickness ratio equal to 20 and 11 layers.
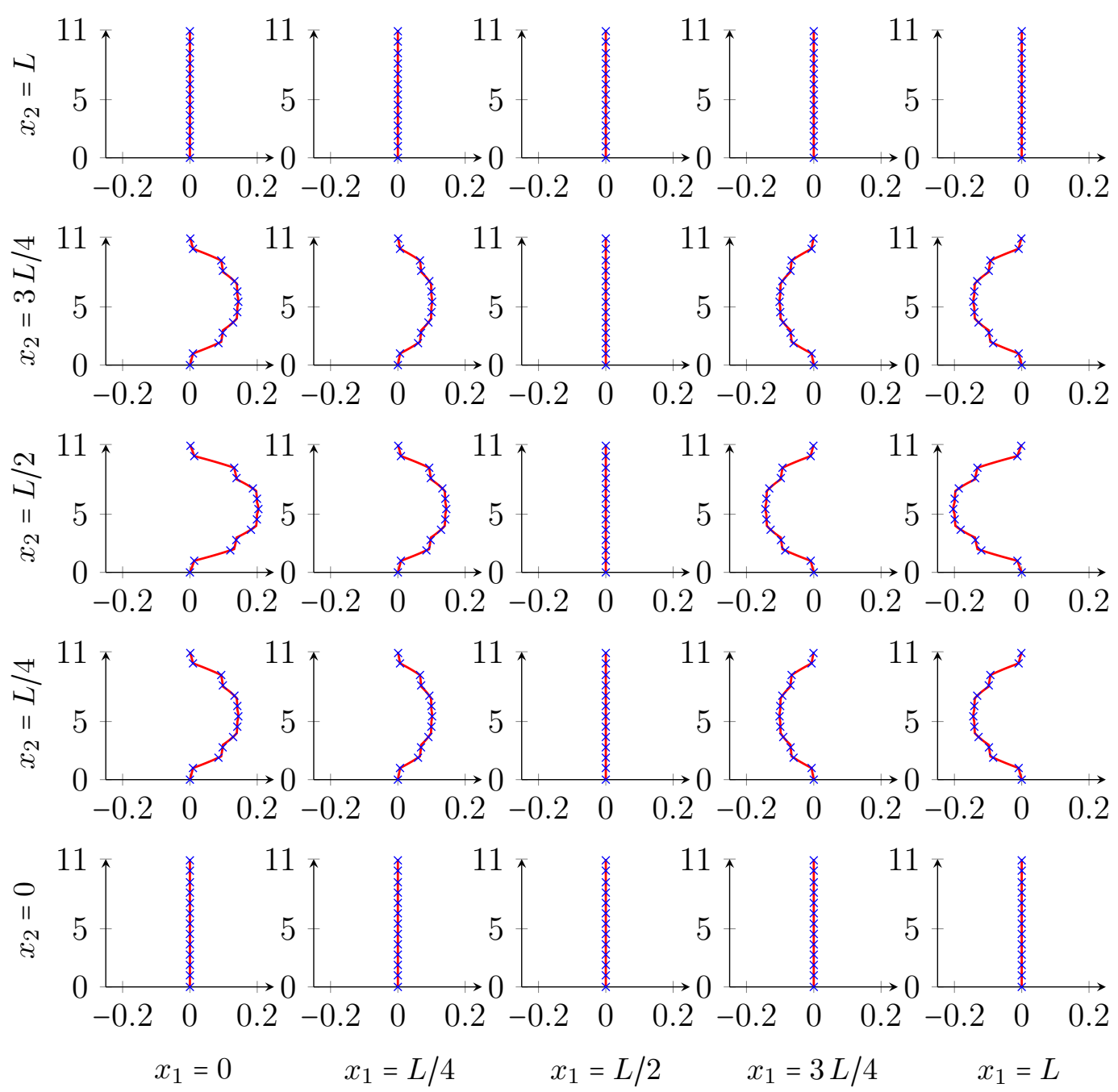

Figure 5: Through-the-thickness $\bar{\sigma}_{13}$ profiles for several in plane sampling points. $L$ represents the total length of the plate, that for this case is $L=220 \mathrm{~mm}$ (being $L=S t$ with $t=11 \mathrm{~mm}$ and $S=20$ ), while the number of layers is 11 (-post-processed solution, $\times$ analytical solution [60]). 

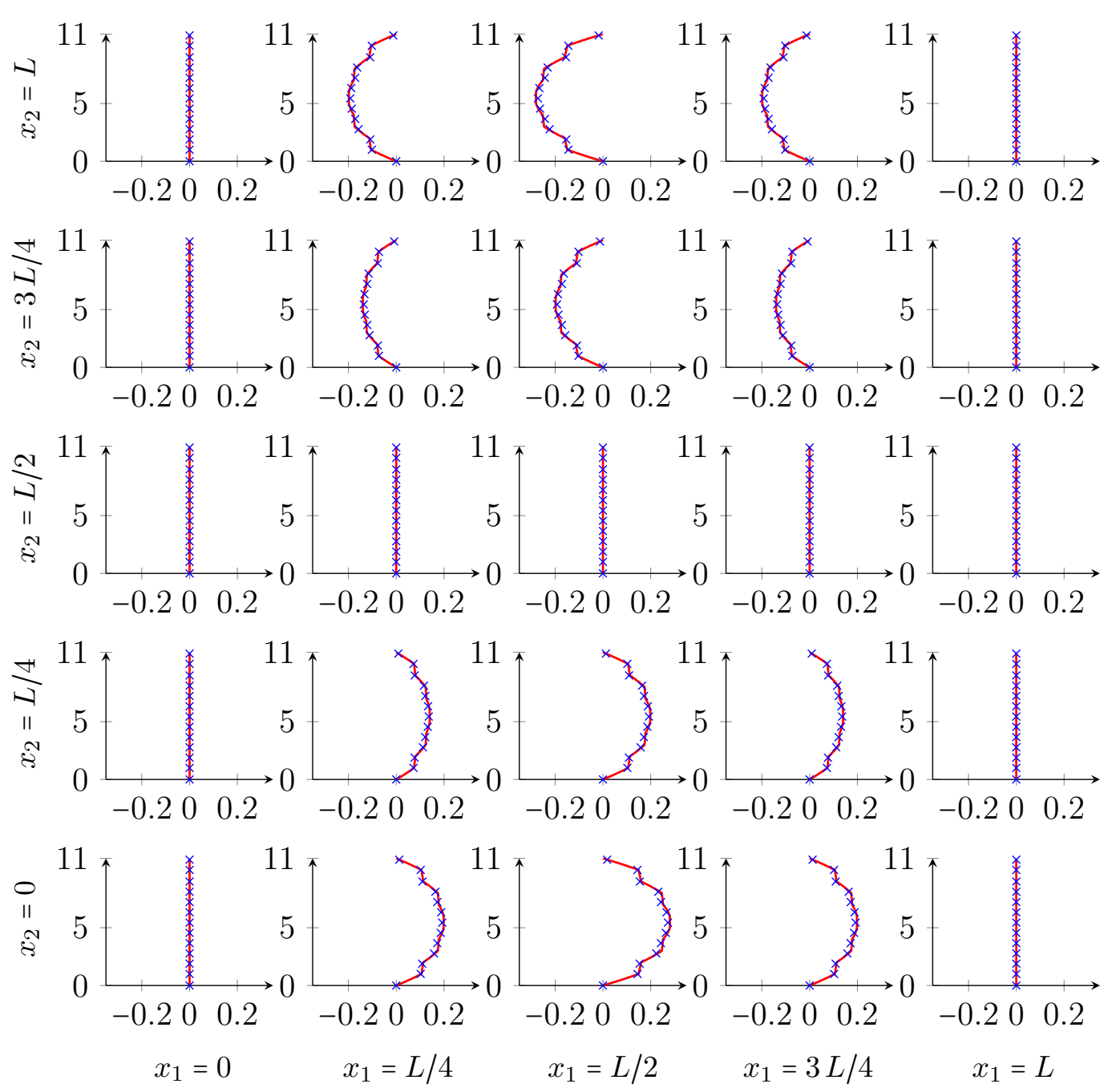

Figure 6: Through-the-thickness $\bar{\sigma}_{23}$ profiles for several in plane sampling points. $L$ represents the total length of the plate, that for this case is $L=220 \mathrm{~mm}$ (being $L=S t$ with $t=11 \mathrm{~mm}$ and $S=20$ ), while the number of layers is 11 (-post-processed solution, $\times$ analytical solution [60]). 

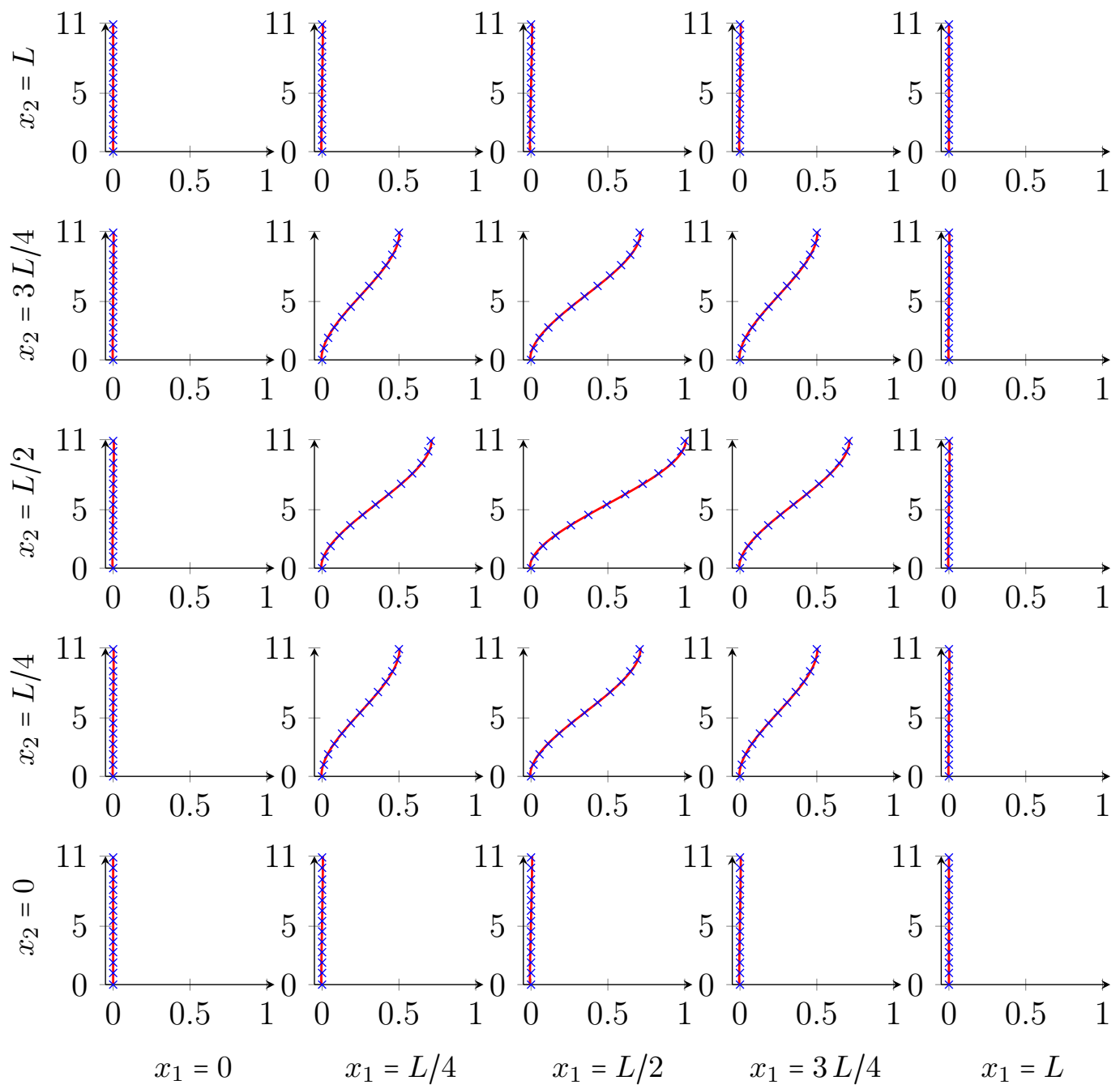

Figure 7: Through-the-thickness $\bar{\sigma}_{33}$ profiles for several in plane sampling points. $L$ represents the total length of the plate, that for this case is $L=220 \mathrm{~mm}$ (being $L=S t$ with $t=11 \mathrm{~mm}$ and $S=20$ ), while the number of layers is 11 (-post-processed solution, $\times$ analytical solution [60]). 


\subsection{Convergence behaviour}

In order to validate the proposed approach in a wider variety of cases, computations with a different ratio between the thickness of the plate and its length are performed respectively for 3, 11, and 33 layers, considering an increasing number of knot spans. Figures 8 and 9 assess the convergence behaviour of the method, adopting the following error definition

$$
\mathrm{e}\left(\sigma_{i j}\right)=\frac{\max \left(\left|\sigma_{i j}^{\text {analytic }}-\sigma_{i j}^{\text {recovered }}\right|\right)}{\max \left(\left|\sigma_{i j}^{\text {analytic }}\right|\right)} .
$$

Note that relation $(22)$ is used only to estimate the error inside the domain to avoid indeterminate forms. Different combinations of degree of approximations have been also considered. A poorer out-of-plane stress approximation is obtained using a degree equal to 4 in every direction, and, in addition, with this choice locking phenomena may occur for increasing values of length-tothickness ratio. Therefore, we conclude that using a degree of approximation equal to 6 in-plane and equal to 4 through the thickness seems to be a reasonable choice to correctly reproduce the 3D stress state. Using instead uniform approximation degrees $p=q=r=6$ does not seem to significantly improve the results (see Figures 8, 9, and Table 2). The post-processing method provides better results for increasing values of length-to-thickness ratio and number of layers and therefore proves to be particularly convenient for very large and thin plates. This is clear since a laminae with a large number of thin layers resembles a plate with average properties. What really stands out looking at the displayed mesh sensitivity results, is the fact that collocation perfectly captures the plates behaviour not only using one element through the thickness but also employing only one knot span in the plane of the plate. 
A single element of degrees $p=q=6$ and $r=4$, comprising a total of $7 \times 7 \times 5$ collocation points, is able to provide for this example maximum percentage errors of $5 \%$ or lower (and of $1 \%$ or lower in the cases of 11 and 33 layers) for $S=30$ or larger.

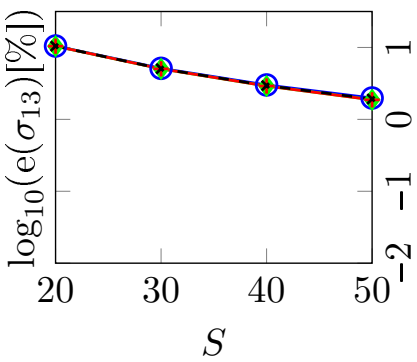

(a) 3 layers

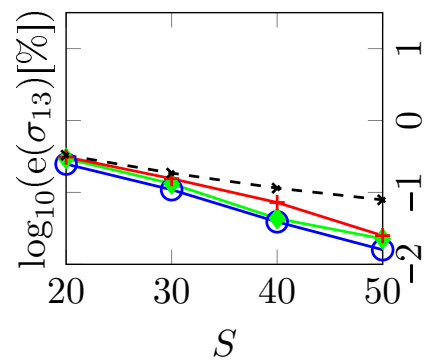

(d) 11 layers

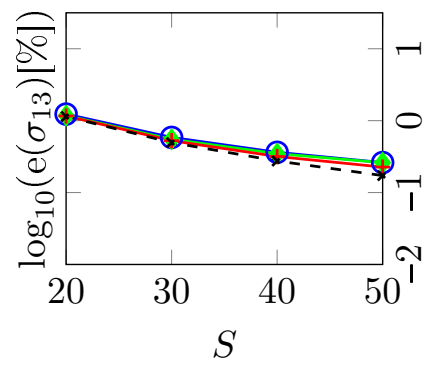

(g) 33 layers

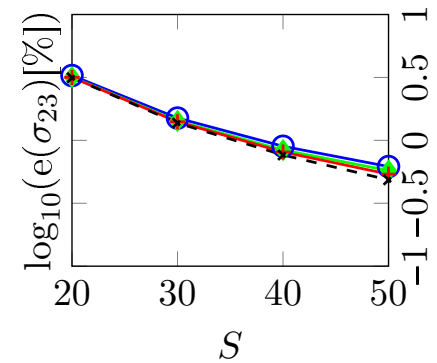

(b) 3 layers

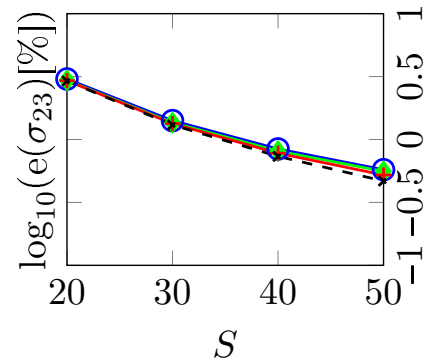

(e) 11 layers

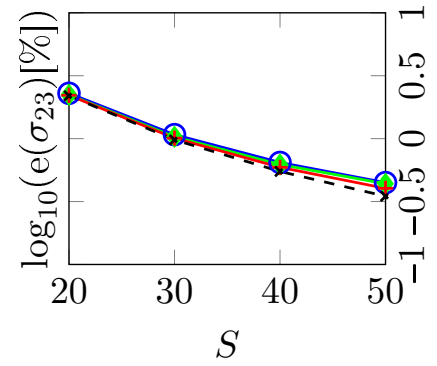

(h) 33 layers

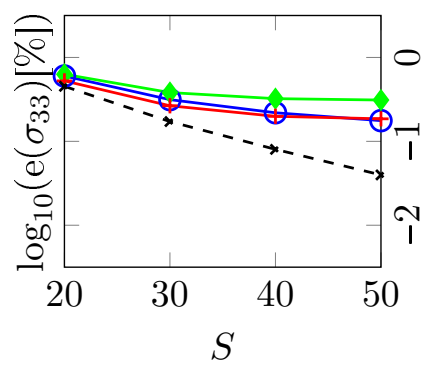

(c) 3 layers

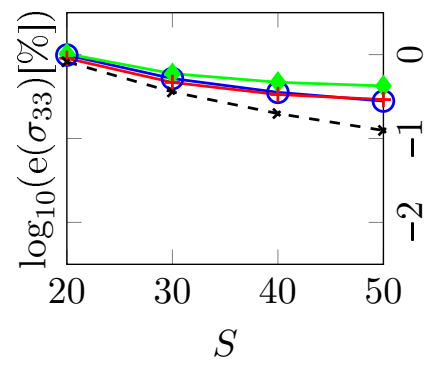

(f) 11 layers

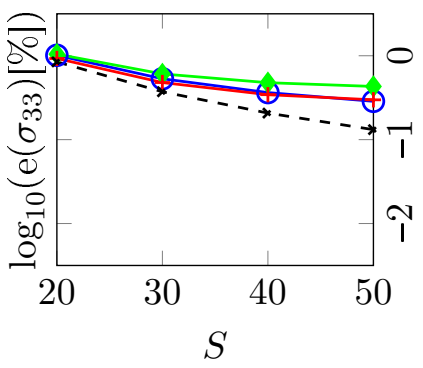

(i) 33 layers

Figure 8: Maximum relative percentage error evaluation at $x_{1}=x_{2}=0.25 L$ for in-plane degree of approximation equal to 6 and out-of-plane degree of approximation equal to 4 . Different length-to-thickness ratios $S$ are investigated for a number of layers equal to 3 , 11 , and 33 ( 1 knot span, $>2$ knot spans, + 4 knot spans, $\times 8$ knot spans). 


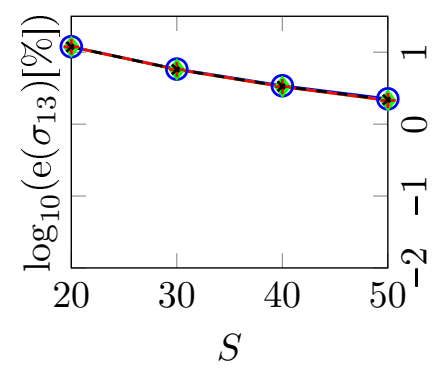

(a) 3 layers

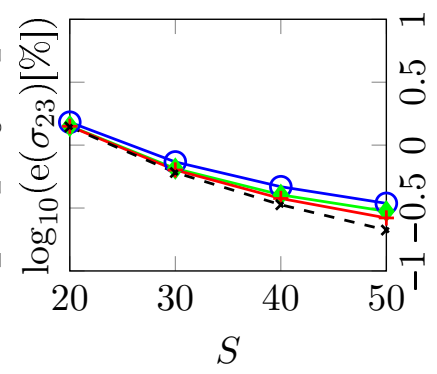

(b) 3 layers

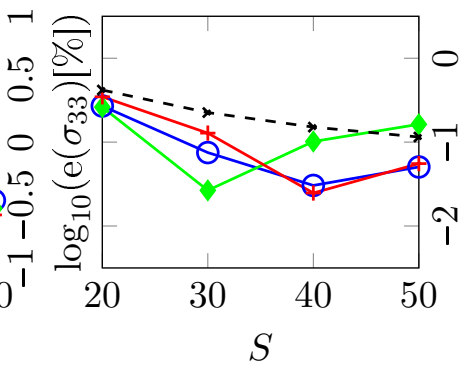

(c) 3 layers

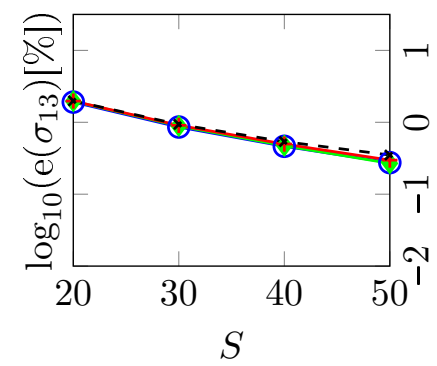

(d) 11 layers

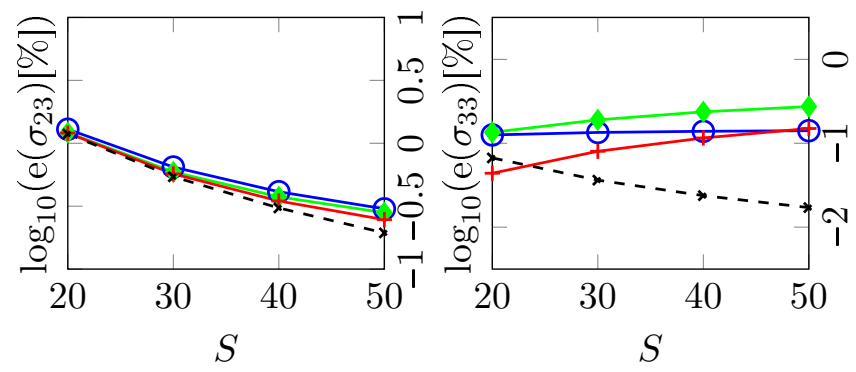

(e) 11 layers

(f) 11 layers

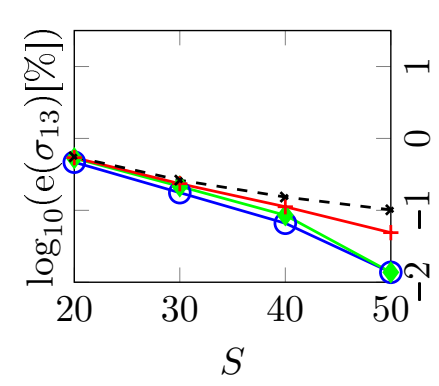

(g) 33 layers

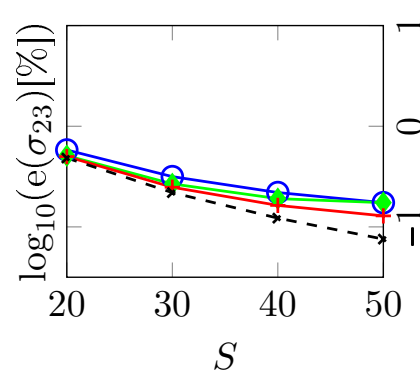

(h) 33 layers

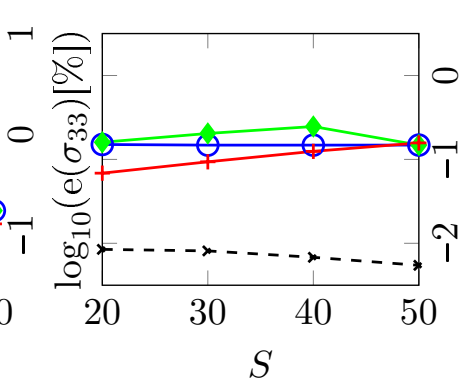

(i) 33 layers

Figure 9: Maximum relative percentage error evaluation at $x_{1}=x_{2}=0.25 \mathrm{~L}$ for degree of approximation equal to 6. Different length-to-thickness ratios $S$ are investigated for a number of layers equal to 3,11 , and 33 ( 1 knot span, 2 knot spans, +4 knot spans, $\times 8$ knot spans). 
Quantitative results are presented in Table 2 for various plate cases, considering a number of layers equal to 11 and 10 collocation points for each in-plane parametric direction. Different number of layers (i.e., 3 and 33) are instead investigated in Appendix A. Increasing length-to-thickness ratios, namely 20, 30, 40, and 50 are considered and the maximum relative error results is reported for a reference point located at $x_{1}=x_{2}=0.25 \mathrm{~L}$. Also different degrees of approximation are investigated. Given these results, we conclude that using an out-of-plane degree of approximation equal to 4 leads to a sufficiently accurate stress state. Furthermore the out-of-plane stress profile reconstruction shows a remarkable improvement for increasing values of number of layers and slenderness parameter $S$.

Table 2: Simply supported composite plate under sinusoidal load with a number of layers equal to 11. Out-of-plane stress state maximum relative error with respect to Pagano's solution [60] at $x_{1}=x_{2}=0.25 \mathrm{~L}$. Comparing the isogeometric collocation-based homogenized single element approach (IGA-C) and the coupled post-processing technique (IGA-C $+\mathrm{PP}$ ) for different approximation degrees.

\begin{tabular}{|c|c|c|c|c|c|c|c|}
\hline \multicolumn{2}{|c|}{ Degree } & \multicolumn{3}{c|}{$p=q=6, r=4$} & \multicolumn{3}{c|}{$p=q=r=6$} \\
\hline \multirow{2}{*}{$\mathbf{S}$} & \multirow{2}{*}{ Method } & $e\left(\sigma_{13}\right)$ & $e\left(\sigma_{23}\right)$ & $e\left(\sigma_{33}\right)$ & $e\left(\sigma_{13}\right)$ & $e\left(\sigma_{23}\right)$ & $e\left(\sigma_{33}\right)$ \\
\cline { 3 - 8 } & & {$[\%]$} & {$[\%]$} & {$[\%]$} & {$[\%]$} & {$[\%]$} & {$[\%]$} \\
\hline \multirow{2}{*}{20} & IGA-C & 97.6 & 56.7 & 6.34 & 96.6 & 56.1 & 6.31 \\
& IGA-C+PP & 0.31 & 2.94 & 0.90 & 1.97 & 1.20 & 0.05 \\
\hline \multirow{2}{*}{30} & IGA-C & 98.7 & 55.6 & 6.36 & 98.3 & 55.4 & 6.34 \\
& IGA-C+PP & 0.16 & 1.34 & 0.47 & 0.91 & 0.57 & 0.08 \\
\hline \multirow{2}{*}{40} & IGA-C & 99.2 & 55.3 & 6.37 & 98.9 & 55.2 & 6.36 \\
& IGA-C+PP & 0.07 & 0.78 & 0.34 & 0.50 & 0.35 & 0.12 \\
\hline \multirow{2}{*}{50} & IGA-C & 99.4 & 55.1 & 6.38 & 99.2 & 55.1 & 6.38 \\
& IGA-C+PP & 0.03 & 0.52 & 0.29 & 0.30 & 0.25 & 0.15 \\
\hline
\end{tabular}




\section{Conclusions}

In this paper we present a new approach to simulate laminated plates characterized by a symmetric distribution of plies. This technique combines a 3D collocation isogeometric analysis with a post-processing step procedure based on equilibrium equations. Since we adopt a single-element appoach, to take into account variation through the plate thickness of the material properties, we average the constitutive behaviour of each layer considering an homogeneized response. Following this simple approach, we showed that acceptable results can be obtained only in terms of displacements and in-plain stresses. Therefore, we propose to perform a post-processing step which requires the shape functions to be highly continuous. This continuity demand is fully granted by typical IGA shape functions. After the post-processing correction is applied, good results are recovered also in terms of out-of-plane stresses, even for very coarse meshes. The post-processing stress-recovery technique is only based on the integration through the thickness of equilibrium equations, and all the required components can be easily computed differentiating the displacement solution. Several numerical tests are carried out to test the sensitivity of the proposed technique to different length-tothickness ratios and number of layers. Regardless of the number of layers, the method gives better results the thinner the composites are. Multiple numbers of alternated layers and sequence of stacks (both even and odd) have been studied in our applications. Neverthless only tests which consider an odd number of layers or an odd disposition of an even number of stacks show good results as expected because considering a homogenized response 
of the material is effective only for symmetric distributions of plies. Further research topics currently under investigation consist in the extension of this approach to more complex problems involving curved geometries and large deformations.

\section{Acknowledgments}

This work was partially supported by Fondazione Cariplo - Regione Lombardia through the project "Verso nuovi strumenti di simulazione super veloci ed accurati basati sull'analisi isogeometrica", within the program RST - rafforzamento.

P. Antolin was partially supported by the European Research council through the H2020 ERC Advanced Grant 2015 n.694515 CHANGE.

\section{Appendix A}

Results in terms of maximum relative error considering a plate with a number of layers equal to 3 and 33 are herein presented for a reference point located at $x_{1}=x_{2}=0.25 \mathrm{~L}$. Increasing length-to-thickness ratios, namely 20, 30, 40, and 50 are investigated for different degrees of approximations (i.e., $p=q=6$ and $r=4$, and $p=q=r=6$ ), using 10 collocation points for each in-plane parametric direction and one element through-the-thickness. 
Table 3: Simply supported composite plate under sinusoidal load with a number of layers equal to 3 . Out-of-plane stress state maximum relative error with respect to Pagano's solution [60] at $x_{1}=x_{2}=0.25 \mathrm{~L}$. Comparing the isogeometric collocation-based homogenized single element approach (IGA-C) and the coupled post-processing technique (IGA-C $+\mathrm{PP}$ ) for different approximation degrees.

\begin{tabular}{|c|c|c|c|c|c|c|c|}
\hline \multicolumn{2}{|c|}{ Degree } & \multicolumn{3}{c|}{$p=q=6, r=4$} & \multicolumn{3}{c|}{$p=q=r=6$} \\
\hline \multirow{2}{*}{$\mathrm{S}$} & \multirow{2}{*}{ Method } & $e\left(\sigma_{13}\right)$ & $e\left(\sigma_{23}\right)$ & $e\left(\sigma_{33}\right)$ & $e\left(\sigma_{13}\right)$ & $e\left(\sigma_{23}\right)$ & $e\left(\sigma_{33}\right)$ \\
\cline { 3 - 8 } & & {$[\%]$} & {$[\%]$} & {$[\%]$} & {$[\%]$} & {$[\%]$} & {$[\%]$} \\
\hline \multirow{2}{*}{20} & IGA-C & 292 & 57.2 & 5.80 & 291 & 57.2 & 5.79 \\
& IGA-C+PP & 10.4 & 3.16 & 0.54 & 11.9 & 1.41 & 0.33 \\
\hline \multirow{3}{*}{30} & IGA-C & 311 & 57.5 & 5.77 & 311 & 57.5 & 5.77 \\
& IGA-C+PP & 5.05 & 1.40 & 0.28 & 5.75 & 0.63 & 0.11 \\
\hline \multirow{3}{*}{40} & IGA-C & 319 & 57.6 & 5.76 & 319 & 57.6 & 5.76 \\
& IGA-C+PP & 2.91 & 0.81 & 0.21 & 3.32 & 0.38 & 0.02 \\
\hline \multirow{2}{*}{50} & IGA-C & 323 & 57.6 & 5.76 & 322 & 57.6 & 5.76 \\
& IGA-C+PP & 1.87 & 0.54 & 0.20 & 2.14 & 0.26 & 0.07 \\
\hline
\end{tabular}

Table 4: Simply supported composite plate under sinusoidal load with a number of layers equal to 33. Out-of-plane stress state maximum relative error with respect to Pagano's solution 60] at $x_{1}=x_{2}=0.25 \mathrm{~L}$. Comparing the isogeometric collocation-based homogenized single element approach (IGA-C) and the coupled post-processing technique (IGA-C $+\mathrm{PP}$ ) for different approximation degrees.

\begin{tabular}{|c|c|c|c|c|c|c|c|}
\hline \multicolumn{2}{|c|}{ Degree } & \multicolumn{3}{|c|}{$p=q=6, r=4$} & \multicolumn{3}{c|}{$p=q=r=6$} \\
\hline \multirow{2}{*}{ S } & \multirow{2}{*}{ Method } & $e\left(\sigma_{13}\right)$ & $e\left(\sigma_{23}\right)$ & $e\left(\sigma_{33}\right)$ & $e\left(\sigma_{13}\right)$ & $e\left(\sigma_{23}\right)$ & $e\left(\sigma_{33}\right)$ \\
\cline { 3 - 8 } & & {$[\%]$} & {$[\%]$} & {$[\%]$} & {$[\%]$} & {$[\%]$} & {$[\%]$} \\
\hline \multirow{2}{*}{20} & IGA-C & 81.6 & 69.7 & 6.33 & 80.7 & 68.9 & 6.33 \\
& IGA-C+PP & 1.16 & 2.21 & 0.93 & 0.54 & 0.50 & 0.07 \\
\hline \multirow{2}{*}{30} & IGA-C & 81.5 & 69.0 & 6.34 & 81.2 & 68.7 & 6.34 \\
& IGA-C+PP & 0.53 & 1.01 & 0.48 & 0.23 & 0.25 & 0.09 \\
\hline \multirow{2}{*}{40} & IGA-C & 81.5 & 68.7 & 6.35 & 81.3 & 68.6 & 6.34 \\
& IGA-C+PP & 0.32 & 0.59 & 0.34 & 0.11 & 0.16 & 0.12 \\
\hline \multirow{2}{*}{50} & IGA-C & 81.6 & 68.6 & 6.35 & 81.4 & 68.5 & 6.35 \\
& IGA-C+PP & 0.23 & 0.40 & 0.30 & 0.05 & 0.13 & 0.16 \\
\hline
\end{tabular}




\section{References}

[1] I. Akkerman, Y. Bazilevs, V.M. Calo, T.J.R. Hughes, and S. Hulshoff. The role of continuity in residual-based variational multiscale modeling of turbulence. Computational Mechanics, 41: 371-378, 2008.

[2] C. Anitescu, Y. Jia, Y.J. Zhang, and T. Rabczuk. An isogeometric collocation method using superconvergent points. Computer Methods in Applied Mechanics and Engineering, 284: 1073-1097, 2015.

[3] F. Auricchio, L. Beirão da Veiga, A. Buffa, C. Lovadina, A. Reali, and G. Sangalli. A fully locking-free isogeometric approach for plane linear elasticity problems: A stream function formulation. Computer Methods in Applied Mechanics and Engineering, 197: 160-172, 2007.

[4] F. Auricchio, L. Beirão da Veiga, T.J.R. Hughes, A. Reali, and G. Sangalli. Isogeometric collocation methods. Mathematical Models and Methods in Applied Sciences, 20: 2075-2107, 2010.

[5] F. Auricchio, L. Beirão da Veiga, C. Lovadina, and A. Reali. The importance of the exact satisfaction of the incompressibility constraint in nonlinear elasticity: mixed FEMs versus NURBS-based approximations. Computer Methods in Applied Mechanics and Engineering, 199: 314$323,2010$.

[6] F. Auricchio, L. Beirão da Veiga, T.J.R. Hughes, A. Reali, and G. Sangalli. Isogeometric collocation for elastostatics and explicit dynamics. 
Computer Methods in Applied Mechanics and Engineering, 249-252: 2$14,2012$.

[7] F. Auricchio, F. Calabrò, T.J.R. Hughes, A. Reali, and G. Sangalli. A simple algorithm for obtaining nearly optimal quadrature rules for NURBS-based isogeometric analysis. Computer Methods in Applied Mechanics and Engineering, 249-252: 15-27, 2012.

[8] F. Auricchio, L. Beirão da Veiga, J. Kiendl, C. Lovadina, and A. Reali. Locking-free isogeometric collocation methods for spatial Timoshenko rods. Computer Methods in Applied Mechanics and Engineering, 263: 113-126, 2013.

[9] G. Balduzzi, S. Morganti, F. Auricchio, and A. Reali. Non-prismatic Timoshenko-like beam model: Numerical solution via isogeometric collocation. Computers and Mathematics with Applications, 74: 1531-1541, 2017.

[10] Y. Bazilevs, M.-C. Hsu, J. Kiendl, R. Wüchner, and K.-U. Bletzinger. 3D simulation of wind turbine rotors at full scale. Part II: Fluid-structure interaction modeling with composite blades. International Journal for Numerical Methods in Fluids, 65: 236-253, 2011.

[11] M.J. Borden, C.V. Verhoosel, M.A. Scott, T.J.R. Hughes, and C.M. Landis. A phase-field description of dynamic brittle fracture. Computer Methods in Applied Mechanics and Engineering, 217-220: 77-95, 2012 . 
[12] A. Buffa, C. de Falco, and G. Sangalli. IsoGeometric Analysis: Stable elements for the $2 \mathrm{~d}$ Stokes equation. International Journal for Numerical Methods in Fluids, 65: 1407-1422, 2011.

[13] J.F. Caseiro, R.A.F. Valente, A. Reali, J. Kiendl, F. Auricchio, and R.J. Alves de Sousa. On the Assumed Natural Strain method to alleviate locking in solid-shell NURBS-based finite elements. Computational Mechanics, 53: 1341-1353, 2014.

[14] H. Casquero, C. Bona-Casas, and H. Gomez. A NURBS-based immersed methodology for fluid-structure interaction. Computer Methods in Applied Mechanics and Engineering, 284: 943-970, 2015.

[15] J.A. Cottrell, T.J.R. Hughes, and A. Reali. Studies of refinement and continuity in isogeometric structural analysis. Computer Methods in Applied Mechanics and Engineering, 196: 4160-4183, 2007.

[16] F. Daghia, S. de Miranda, F. Ubertini, and E. Viola. A hybrid stress approach for laminated composite plates within the First-order Shear Deformation Theory. International Journal of Solids and Structures, 45: 1766-1787, 2008.

[17] L. Beirão da Veiga, C. Lovadina, and A. Reali. Avoiding shear locking for the Timoshenko beam problem via isogeometric collocation methods. Computer Methods in Applied Mechanics and Engineering, 241-244: 38$51,2012$.

[18] C. de Boor. A Practical Guide to Splines. Springer, 1978. 
[19] S. de Miranda and F. Ubertini. Recovery of consistent stresses for compatible finite elements. Computer Methods in Applied Mechanics and Engineering, 191: 1595-1609, 2002.

[20] S. Demko. On the Existence of Interpolating Projections onto Spline Spaces. Journal of Approximation Theory, 43: 151-156, 1985.

[21] L. De Lorenzis, J.A. Evans, T.J.R. Hughes, and A. Reali. Isogeometric collocation: Neumann boundary conditions and contact. Computer Methods in Applied Mechanics and Engineering, 284: 21-54, 2015.

[22] R.P. Dhote, H. Gomez, R.N.V. Melnik, and J. Zu. Isogeometric analysis of a dynamic thermomechanical phase-field model applied to shape memory alloys. Computational Mechanics, 53: 1235-1250, 2014.

[23] J.-E. Dufour, P. Antolin, G. Sangalli, F. Auricchio, and A. Reali. A costeffective isogeometric approach for composite plates based on a stress recovery procedure. Composites Part B, 138: 12-18, 2018.

[24] T. Elguedj, and T.J.R. Hughes. Isogeometric analysis of nearly incompressible large strain plasticity. Computer Methods in Applied Mechanics and Engineering, 268: 388-416, 2014.

[25] J.J. Engblom and O.O. Ochoa. Through-the-thickness stress predictions for laminated plates of advanced composite materials. International Journal for Numerical Methods in Engineering, 21: 1759-1776, 1985.

[26] F. Fahrendorf, L. De Lorenzis, and H. Gomez. Reduced integration at 
superconvergent points in isogeometric analysis. Computer Methods in Applied Mechanics and Engineering, 328: 390-410, 2018.

[27] A. Farzam, and B. Hassani. A new efficient shear deformation theory for FG plates with in-plane and through-thickness stiffness variations using isogeometric approach. Mechanics of Advanced Materials and Structures, 0: 1-14, 2018.

[28] R.F. Gibson. Principles of Composite Material Mechanics. McGraw-Hill, 1994.

[29] H. Gomez, V.M. Calo, Y. Bazilevs, and T.J.R. Hughes. Isogeometric analysis of the Cahn-Hilliard phase-field model. Computer Methods in Applied Mechanics and Engineering, 197: 4333-4352, 2008.

[30] H. Gomez, T.J.R. Hughes, X. Nogueira, and V.M. Calo. Isogeometric analysis of the isothermal Navier-Stokes-Korteweg equations. Computer Methods in Applied Mechanics and Engineering, 199: 1828-1840, 2010.

[31] H. Gomez, A. Reali, and G. Sangalli. Accurate, effcient, and (iso)geometrically flexible collocation methods for phase-field models. Journal of Computational Physics, 153-171, 2014.

[32] Y. Guo, A.P. Nagy, and Z. Gürdal. A layerwise theory for laminated composites in the framework of isogeometric analysis. Composite Structures, 107: 447-457, 2014.

[33] Y. Guo and M. Ruess. A layerwise isogeometric approach for NURBS- 
derived laminate composite shells. Composite Structures, 124: 300-309, 2015 .

[34] Z. Hashin. Theory of fiber reinforced materials. Technical Report NASACR-1974, 1972.

[35] M.-C. Hsu, D. Kamensky, F. Xu, J. Kiendl, C. Wang, M.C.H. Wu, J. Mineroff, A. Reali, Y. Bazilevs, and M.S. Sacks. Dynamic and fluid-structure interaction simulations of bioprosthetic heart valves using parametric design with T-splines and Fung-type material models. Computational Mechanics, 55: 1211-1225, 2015.

[36] T.J.R. Hughes, J.A. Cottrell, and Y. Bazilevs. Isogeometric analysis: CAD, finite elements, NURBS, exact geometry and mesh refinement. Computer Methods in Applied Mechanics and Engineering, 194: 41354195, 2005.

[37] T.J.R. Hughes, A. Reali, and G. Sangalli. Duality and unified analysis of discrete approximations in structural dynamics and wave propagation: Comparison of $p$-method finite elements with $k$-method NURBS. Computer Methods in Applied Mechanics and Engineering, 197: 4104-4124, 2008 .

[38] T.J.R. Hughes, A. Reali, and G. Sangalli. Efficient quadrature for NURBS-based isogeometric analysis. Computer Methods in Applied Mechanics and Engineering, 199: 301-313, 2010.

[39] T.J.R. Hughes, J.A. Evans, and A. Reali Finite element and NURBS approximations of eigenvalue, boundary-value, and initial-value prob- 
lems. Computer Methods in Applied Mechanics and Engineering, 272: 290-320, 2014.

[40] R.W. Johnson. A B-spline collocation method for solving the incompressible Navier-Stokes equations using an ad hoc method: The Boundary Residual method. Computers \&f Fluids, 34: 121-149, 2005.

[41] R.M. Jones. Mechanics of Composite Materials. Second Edition, Taylor E Francis, 1999.

[42] H. Kapoor, R.K. Kapania, and S.R. Soni. Interlaminar stress calculation in composite and sandwich plates in NURBS Isogeometric finite element analysis. Composite Structures, 106: 537-548, 2013.

[43] J. Kiendl, K.-U. Bletzinger, J. Linhard, and R. Wüchner. Isogeometric shell analysis with Kirchhoff-Love elements. Computer Methods in Applied Mechanics and Engineering, 198: 3902-3914, 2009.

[44] J. Kiendl, F. Auricchio, L. Beirão da Veiga, C. Lovadina, and A. Reali. Isogeometric collocation methods for the Reissner-Mindlin plate problem. Computer Methods in Applied Mechanics and Engineering, 284: 489-507, 2015.

[45] J. Kiendl, F. Auricchio, T.J.R. Hughes, and A. Reali. Singlevariable formulations and isogeometric discretizations for shear deformable beams. Computer Methods in Applied Mechanics and Engineering, 284: 988-1004, 2015.

[46] J. Kiendl, E. Marino, and L. De Lorenzis. Isogeometric collocation 
for the Reissner-Mindlin shell problem. Computer Methods in Applied Mechanics and Engineering, 325: 645-665, 2017.

[47] J. Kiendl, F. Auricchio, and A. Reali. A displacement-free formulation for the Timoshenko beam problem and a corresponding isogeometric collocation approach. Meccanica, 53: 1403-1413, 2018.

[48] R. Kruse, N. Nguyen-Thanh, L. De Lorenzis, and T.J.R. Hughes. Isogeometric collocation for large deformation elasticity and frictional contact problems. Computer Methods in Applied Mechanics and Engineering, 296: 73-112, 2015.

[49] S. Lipton, J.A. Evans, Y. Bazilevs, T. Elguedj, and T.J.R. Hughes. Robustness of isogeometric structural discretizations under severe mesh distortion. Computer Methods in Applied Mechanics and Engineering, 199: 357-373, 2010.

[50] J. Liu, H. Gomez, J.A. Evans, T.J.R. Hughes, and C.M. Landis. Functional entropy variables: A new methodology for deriving thermodynamically consistent algorithms for complex fluids, with particular reference to the isothermal Navier-Stokes-Korteweg equations. Journal of Computational Physics, 248: 47-86, 2013.

[51] G. Lorenzo, T.J.R. Hughes, P. Dominguez-Frojan, A. Reali, and H. Gomez. Computer simulations suggest that prostate enlargement due to benign prostatic hyperplasia mechanically impedes prostate cancer growth. Proceedings of the National Academy of Sciences of the United States of America, In press (2018). 
[52] C. Manni, A. Reali, and H. Speleers. Isogeometric collocation methods with generalized B-splines. Computer and Mathematics with Applications, 70: 1659-1675, 2015.

[53] E. Marino. Isogeometric collocation for three-dimensional geometrically exact shear-deformable beams. Computer and Mathematics with Applications, 307: 383-410, 2016.

[54] E. Marino. Locking-free isogeometric collocation formulation for threedimensional geometrically exact shear-deformable beams with arbitrary initial curvature. Computer and Mathematics with Applications, 324: 546-572, 2017.

[55] E. Marino, J. Kiendl, and L. De Lorenzis. Explicit isogeometric collocation for the dynamics of three-dimensional beams undergoing finite motions. Computer and Mathematics with Applications, 343: 530-549, 2019 .

[56] F. Maurin, F. Greco, L. Coox, D. Vandepitte, and W. Desmet. Isogeometric collocation for Kirchhoff-Love plates and shells. Computer Methods in Applied Mechanics and Engineering, 329: 396-420, 2018.

[57] M. Montardini, G. Sangalli, and L. Tamellini. Optimal-order isogeometric collocation at Galerkin superconvergent points. Computer Methods in Applied Mechanics and Engineering, 316: 741-757, 2017.

[58] S. Morganti, F. Auricchio, D.J. Benson, F.I. Gambarin, S. Hartmann, T.J.R. Hughes, and A. Reali. Patient-specific isogeometric structural 
analysis of aortic valve closure. Computer Methods in Applied Mechanics and Engineering, 284: 508-520, 2015.

[59] S. Morganti, C. Callari, F. Auricchio, and A. Reali. Mixed isogeometric collocation methods for the simulation of poromechanics problems in 1D. Meccanica, 53: 1441-1454, 2018.

[60] N.J. Pagano. Exact Solutions for Rectangular Bidirectional Composites and Sandwich Plates. Journal of Composite Materials, 4: 20-34, 1970.

[61] G.S. Pavan, K.S. Nanjunda Rao. Bending analysis of laminated composite plates using isogeometric collocation method. Composite Structures, 176: 715-728, 2017.

[62] L. Piegl and W. Tiller. The NURBS Book (2nd Ed.). Springer, 1997.

[63] C.W. Pryor, and R.M. Barker. A finite-element analysis including transverse shear effects for applications to laminated plates. AIAA Journal, 9: 912-917, 1971.

[64] A. Reali, and H. Gomez. An isogeometric collocation approach for Bernoulli-Euler beams and Kirchhoff plates. Computer Methods in Applied Mechanics and Engineering, 284: 623-636, 2015.

[65] J.N. Reddy. Mechanics of Laminated Composite Plates and Shells: Theory and Analysis (2nd Ed.). CRC Press, 2003.

[66] J.J.C. Remmers, C.V. Verhoosel, and R. de Borst. Isogeometric analysis for modelling of failure in advanced composite materials. In S. R. Hallett, \& P. P. C. (Eds.), Numerical Modelling of Failure in Advanced 
Composite Materials (1 ed., pp. 309-329). (Woodhead Publishing Series in Composites Science and Engineering), 2015.

[67] G. Sangalli, and M. Tani. Matrix-free weighted quadrature for a computationally efficient isogeometric k-method. Computer Methods in Applied Mechanics and Engineering, 338: 117-133, 2018.

[68] D. Schillinger, J.A. Evans, A. Reali, M.A. Scott, and T.J.R. Hughes. Isogeometric collocation: Cost comparison with Galerkin methods and extension to adaptive hierarchical NURBS discretizations. Computer Methods in Applied Mechanics and Engineering, 267: 170-232, 2013.

[69] P. Shi-Dong, W. Lin-Zhi, and S. Yu-Guo. Transverse shear modulus and strength of honeycomb cores. Composite Structures, 84: 369-374, 2018 .

[70] S. Sridharan. Delamination Behaviour of Composites. Woodhead Publishing Series in Composites Science and Engineering, 2008.

[71] C.T. Sun and Sijian Li Three-Dimensional Effective Elastic Constants for Thick Laminates. Journal of Composite Materials, 22: 629-639, 1988.

[72] C.H. Thai, H. Nguyen-Xuan, S.P.A. Bordas, N. Nguyen-Thanh, and T. Rabczuk. Isogeometric analysis of laminated composite plates using the higher-order shear deformation theory. Mechanics of Advanced Materials and Structures, 22: 451-469, 2015. 
[73] F. Ubertini. Patch recovery based on complementary energy. International Journal for Numerical Methods in Engineering, 59: 1501-1538, 2004 .

[74] J.R. Vinson and R.L. Sierakowski. The Behavior of Structures Composed of Composite Materials. Springer, 1986.

[75] O. Weeger, S.-K. Yeung, and M.L. Dunn. Isogeometric collocation methods for Cosserat rods and rod structures. Computer Methods in Applied Mechanics and Engineering, 316: 100-122, 2017.

[76] O. Weeger, B. Narayanana, L. De Lorenzis, J. Kiendl, M.L. Dunn. An isogeometric collocation method for frictionless contact of Cosserat rods. Computer Methods in Applied Mechanics and Engineering, 321: 361$382,2017$.

[77] O. Weeger, S.-K. Yeung, and M.L. Dunn. Fully isogeometric modeling and analysis of nonlinear 3D beams with spatially varying geometric and material parameters. Computer Methods in Applied Mechanics and Engineering, 342: 95-115, 2018. 